\title{
The homotopy type of the space of algebraic loops on a toric variety
}

\author{
Andrzej Kozlowski* and Kohhei Yamaguchi ${ }^{\dagger}$
}

\begin{abstract}
We investigate the homotopy type of the space of tuples of polynomials inducing base-point preserving algebraic maps from the circle $S^{1}$ to a toric variety $X_{\Sigma}$. In particular, we prove a homotopy stability result for this space by combining the Vassiliev spectral sequence [27] and the scanning map [26].
\end{abstract}

\section{Introduction}

For two topological spaces $X$ and $Y$ with base-points, let $\operatorname{Map}^{*}(X, Y)$ denote the space of all continuous based maps (i.e. base-point preserving maps) $f: X \rightarrow Y$ with compact-open topology. When these two spaces have some additional structure, e.g. that of a complex or symplectic manifold or an algebraic variety, it is natural to consider the subspace $\mathcal{S}(X, Y) \subset \operatorname{Map}^{*}(X, Y)$ of all based maps $f$ which preserve this structure and to ask whether the inclusion map $i: \mathcal{S}(X, Y) \rightarrow \operatorname{Map}^{*}(X, Y)$ is a homotopy or homology equivalence up to some dimension. Early examples of this type of phenomenon can be found in [9]. In many cases of interest the infinite dimensional space $\mathcal{S}(X, Y)$ has a filtration by finite dimensional subspaces, given by some kind of "degree of maps", and the topology of these finite dimensional spaces approximates the topology of the entire space of continuous maps; the approximation becoming more accurate as the degree increases. A great deal of attention has been devoted to problems of the above kind in the case where

*Institute of Applied Mathematics and Mechanics, University of Warsaw, Banacha 2, 02-097 Warsaw, Poland (E-mail: akoz@mimuw.edu.pl)

$\dagger$ Department of Mathematics, University of Electro-Communications, Chofu, Tokyo 182-8585, Japan (E-mail: kohhe@im.uec.ac.jp); The second author is supported by JSPS KAKENHI Grant Number 18K03295.

2010 Mathematics Subject Clasification. Primary 55P10; Secondly 55R80, 55P35, 14M25. 
the "additional structure" in question is the structure of a complex manifold (so that the structure preserving maps are holomorphic maps). The first explicit result of this kind seems to have been the following theorem of Segal:

Theorem 1.1 (G. Segal, [26]). If $d \in \mathbb{N}$, the inclusion map

$$
i_{d}: \operatorname{Hol}_{d}^{*}\left(S^{2}, \mathbb{C P}^{n}\right) \rightarrow \operatorname{Map}_{d}^{*}\left(S^{2}, \mathbb{C P}^{n}\right)=\Omega_{d}^{2} \mathbb{C P}^{n} \simeq \Omega^{2} S^{2 n+1}
$$

is a homotopy equivalence through dimension $(2 n-1) d-1$. Here, we denote by $\mathbb{N}$ the space of all positive integers and the space $\operatorname{Hol}_{d}^{*}\left(S^{2}, \mathbb{C P}^{n}\right)$ (resp. $\Omega_{d}^{2} \mathbb{C P}^{n}$ ) denotes the space consisting of all based holomorphic (resp. based continuous) maps $f: S^{2} \rightarrow \mathbb{C P}^{n}$ of degree $d \mathbb{1}$

Remark 1.2. Recall that a map $g: V \rightarrow W$ is called a homology (resp. homotopy) equivalence through dimension $N$ if the induced homomorphism $g_{*}: H_{k}(V ; \mathbb{Z}) \rightarrow H_{k}(W ; \mathbb{Z})$ (resp. $g_{*}: \pi_{k}(V) \rightarrow \pi_{k}(W)$ ) is an isomorphism for all $k \leq N$.

Segal conjectured that this theorem should have analogues for holomorphic maps from the Riemann sphere $S^{2}$ (or even any compact Riemann surface) to various complex manifolds $Y$. He also suggested that some analogues may exists for higher dimensional complex manifolds in place of $S^{2}$. All of Segal's conjectures have been shown to be true, although no fully satisfactory general explanation of these phenomena has been found.

Guest 11] was the first one to consider Segal's problem for the space of holomorphic maps from $S^{2}$ to a compact toric variety. His result was very general but the stability dimension he obtained was rather low. A much better stability bound was obtained by Mostovoy and Munguia-Villanueva [24]. Using methods developed by Vassiliev and Mostovoy [23], they were able to obtain a Segal type theorem for the case of spaces of holomorphic maps from $\mathbb{C P}^{n}$ for $n \geq 1$ to complex compact toric varieties. Although their method works with arbitrary $\mathbb{C P}^{n}$ as source, for technical reasons the target space has to be compact. That leaves out some interesting toric varieties for which Segal's type theorems still hold (see for example [17]). By replacing Mostovoy's approach (which relies on the Stone-Weierstrass Theorem) we were able to prove the Mostovoy and Munguia-Villanueva result for a larger class of target varieties but with $\mathbb{C P}^{1}=S^{2}$ as the source space and this is stated as follows.

Theorem $1.3([20])$. Let $X_{\Sigma}$ be a simply connected non-singular toric variety associated to the fan $\Sigma$ such that the condition (2.14.1) (see below) is satisfied ${ }^{2}$ Then if $D=\left(d_{1}, \cdots, d_{r}\right) \in \mathbb{N}^{r}$ and $\sum_{k=1}^{r} d_{k} \boldsymbol{n}_{k}=\mathbf{0}_{r}$, the inclusion

\footnotetext{
${ }^{1}$ The stablization dimension $(2 n-1) d-1$ obtained by Segal is not optimal for $n \geq 2$. The optimal dimension is $(2 n-1)(d+1)-1$ (see [17]).

${ }^{2}$ If $X_{\Sigma}$ is compact, the condition (2.14,1) is always satisfied (see Remark 2.8).
} 
map

$$
i_{D, h o l}: \operatorname{Hol}_{D}^{*}\left(S^{2}, X_{\Sigma}\right) \stackrel{\subset}{\longrightarrow} \Omega_{D}^{2} X_{\Sigma}
$$

is a homotopy equivalence through dimension $d_{*}(D, \Sigma)$ if $r_{\min }(\Sigma) \geq 3$ and a homology equivalence through dimension $d_{*}(D, \Sigma)=d_{\min }-2$ if $r_{\min }(\Sigma)=2$.

Here, $\Omega_{D}^{2} X_{\Sigma}$ (resp. $\operatorname{Hol}_{D}^{*}\left(S^{2}, X_{\Sigma}\right)$ ) denotes the space of based continuous (resp. based holomorphic) maps from $S^{2}$ to $X_{\Sigma}$ of degree $D$, the number $r_{\min }(\Sigma)$ is defined in 2.24) and $d_{*}(D, \Sigma)$ is the number given by

$$
d_{*}(D, \Sigma)=\left(2 r_{\min }(\Sigma)-3\right) d_{\min }-2, \text { where } d_{\min }=\min \left\{d_{1}, \cdots, d_{r}\right\}
$$

In his seminal work Segal observed that a real analogue of his theorem also holds. In fact, it was this real case that inspired the interest in the entire area. He considered the space of real rational maps, that is pairs of monic polynomials of the same degree with real coefficients and without common (complex) roots (see [26, Proposition 1.4]).

Later in [13], 21] and [1, a different 'real version' of Seqal's theory was introduced; it involves looking at tuples of real polynomials of the same degree without common real roots. One such case was considered in [14, where the analogue of the Mostovoy and Munguia-Villanueva theorem was proved for real rational maps from $\mathbb{R P}^{n}$ to a compact complex toric variety. The method used in it was the same as in [24] and it was limited only to case of compact toric varieties. By imposing a condition involving common real roots rather than complex ones, one can also study spaces of rational maps from $S^{1}$ to complex toric varieties.

An important difference between them is that tuples of polynomials without common real roots no longer correspond to algebraic maps. For example, a pair $(p(z), q(z))$ of monic polynomials of the same degree, without a common real root defines a unique rational (algebraic) map $S^{1} \rightarrow \mathbb{C P}^{1}$ but by multiplying both polynomials by the same monic polynomial without real roots, we obtain the same rational map. Thus it is necessary to distinguish between spaces of tuples of polynomials and that of rational maps - their image in the space of continuous maps.

Now we can state the main result of this paper (precise definitions of all the terms used will be given in \$2).

Theorem 1.4 (Theorem [2.16, Theorem [2.20). Let $D=\left(d_{1}, \cdots, d_{r}\right) \in \mathbb{N}^{r}$ be an r-tuple of positive integers and let $X_{\Sigma}$ be a simply connected nonsingular toric variety associated to the fan $\Sigma$ such that the condition 2.14.1) is satisfied. Then the natural map

$$
j_{D}: \operatorname{Pol}_{D}^{*}\left(S^{1}, X_{\Sigma}\right) \rightarrow \Omega U\left(\mathcal{K}_{\Sigma}\right) \simeq \Omega \mathcal{Z}_{\mathcal{K}_{\Sigma}}
$$


is a homotopy equivalence through dimension $d(D, \Sigma)$, where $d_{\text {min }}$ denotes the positive integer $d_{\min }=\min \left\{d_{1}, \cdots, d_{r}\right\}$ and the number $d(D, \Sigma)$ is given by

$$
d(D, \Sigma)=2\left(r_{\min }(\Sigma)-1\right) d_{\min }-2 .
$$

Here the space $U\left(\mathcal{K}_{\Sigma}\right)$ (resp. $\mathcal{Z}_{\mathcal{K}_{\Sigma}}$ ) is the complement $U\left(\mathcal{K}_{\Sigma}\right)$ of the coordinate subspaces of type $\mathcal{K}_{\Sigma}$ (resp. the moment-angle complex of the underlying simplicial complex $\left.\mathcal{K}_{\Sigma}\right)$ and $\operatorname{Pol}_{D}^{*}\left(S^{1}, X_{\Sigma}\right)$ denotes the space of $r$-tuples $\left(p_{1}(z), \ldots, p_{r}(z)\right) \in \mathbb{C}[z]^{r}$ of monic polynomials of degrees $d_{1}, \ldots, d_{r}$ satisfying the condition (2.19.1). The image of this space under $j_{D}$ is contained in the space $\operatorname{Alg}^{*}\left(S^{1}, X_{\Sigma}\right)$ of based algebraic loops on $X_{\Sigma}$.

Corollary 1.5 (Corollary 2.17, Corollary 2.21). Under the same assumptions as Theorem 1.4, there is a natural map

$$
i_{D}: \operatorname{Pol}_{D}^{*}\left(S^{1}, X_{\Sigma}\right) \rightarrow \Omega X_{\Sigma}
$$

which induces an isomorphism on homotopy groups

$$
\left(i_{D}\right)_{*}: \pi_{k}\left(\mathrm{Pol}_{D}^{*}\left(S^{1}, X_{\Sigma}\right)\right) \stackrel{\cong}{\longrightarrow} \pi_{k}\left(\Omega X_{\Sigma}\right) \cong \pi_{k+1}\left(X_{\Sigma}\right)
$$

for any $2 \leq k \leq d(D, \Sigma)$.

This paper is organized as follows. In $\$ 2$ we recall some basic facts about toric varieties and the definitions needed to give precise statements of our results. We also establish the notation that will be followed in the rest of the paper and then give full statements of all the main results (Theorem 2.16, Corollary 2.17, Theorem 2.20, Corollary 2.21, Corollary 2.18, Corollary 2.22), which will be proved in \$9. We also consider some example (Example 2.25). In 93 we recall several definitions and known results from toric topology. In 84 , we recall the definitions of the non-degenerate simplicial resolution and the associated truncated simplicial resolutions. In 95, we construct the Vassiliev spectral sequence and compute its $E_{1}$-terms. In 6 we define stabilization maps and prove the homotopy stability (Theorem 6.5, Theorem 6.6). Moreover, we give the definition of the map $j_{D}$ when $\sum_{k=1}^{r} d_{k} \boldsymbol{n}_{k} \neq \mathbf{0}_{n}$. In 97 we recall the stable horizontal scanning map and prove that it is a homotopy equivalence (Theorem [7.8). In 88 we give the proof of stability result (Theorem 8.2 ) by using the stable scanning maps. Finally in $\$ 9$ we give the proofs of the main results of this paper.

\section{Basic definitions and the main results}

In this section we shall recall several basic definitions and facts for describing the main results. 
Fans and toric varieties A convex rational polyhedral cone $\sigma$ in $\mathbb{R}^{n}$ is a subset of $\mathbb{R}^{n}$ of the form

$$
\sigma=\operatorname{Cone}(S)=\operatorname{Cone}\left(\boldsymbol{m}_{1}, \cdots, \boldsymbol{m}_{s}\right)=\left\{\sum_{k=1}^{s} \lambda_{k} \boldsymbol{m}_{k}: \lambda_{k} \geq 0 \text { for any } k\right\}
$$

for a finite set $S=\left\{\boldsymbol{m}_{k}\right\}_{k=1}^{s} \subset \mathbb{Z}^{n} 3$ A convex rational polyhedral cone $\sigma$ is called strongly convex if $\sigma \cap(-\sigma)=\left\{\mathbf{0}_{n}\right\}$, and its $\operatorname{dimension} \operatorname{dim} \sigma$ is the dimension of the smallest subspace in $\mathbb{R}^{n}$ which contains $\sigma$. A face $\tau$ of $\sigma$ is a subset $\tau \subset \sigma$ of the form

$$
\tau=\sigma \cap\left\{\boldsymbol{x} \in \mathbb{R}^{n}: L(\boldsymbol{x})=0\right\}
$$

for some linear form $L$ on $\mathbb{R}^{n}$, such that $L(\boldsymbol{x}) \geq 0$ for any $\boldsymbol{x} \in \sigma$. If $\left\{k: L\left(\boldsymbol{m}_{k}\right)=0,1 \leq k \leq s\right\}=\left\{i_{1}, \cdots, i_{t}\right\}$, we easily see that $\tau=$ Cone $\left(\boldsymbol{m}_{i_{1}}, \cdots, \boldsymbol{m}_{i_{t}}\right)$. Thus, a face $\tau$ is also a strongly convex rational polyhedral cone if $\sigma$ is so.

A finite collection $\Sigma$ of strongly convex rational polyhedral cones in $\mathbb{R}^{n}$ is called $a$ fan in $\mathbb{R}^{n}$ if every face $\tau$ of $\sigma \in \Sigma$ belongs to $\Sigma$ and the intersection of any two elements of $\Sigma$ is a face of each.

An $n$ dimensional irreducible normal variety $X$ (over $\mathbb{C}$ ) is called a toric variety if it has a Zariski open subset $\mathbb{T}_{\mathbb{C}}^{n}=\left(\mathbb{C}^{*}\right)^{n}$ and the action of $\mathbb{T}_{\mathbb{C}}^{n}$ on itself extends to an action of $\mathbb{T}_{\mathbb{C}}^{n}$ on $X$. The most significant property of a toric variety is the fact that it is characterized up to isomorphism entirely by its associated fan $\Sigma$. We denote by $X_{\Sigma}$ the toric variety associated to a fan $\Sigma$ (see [7] for the details).

Since the fan of $\mathbb{T}_{\mathbb{C}}^{n}$ is $\left\{\mathbf{0}_{n}\right\}$ and the case $X_{\Sigma}=\mathbb{T}_{\mathbb{C}}^{n}$ is trivial, we always assume that any fan $\Sigma$ in $\mathbb{R}^{n}$ satisfies the condition $\left\{\mathbf{0}_{n}\right\} \varsubsetneqq \Sigma$.

Definition 2.1. Let $\Sigma$ be a fan in $\mathbb{R}^{n}$ such that $\left\{\mathbf{0}_{n}\right\} \varsubsetneqq \Sigma$ and let

$$
\Sigma(1)=\left\{\rho_{1}, \cdots, \rho_{r}\right\}
$$

denote the set of all one dimensional cones in $\Sigma$. For each integer $1 \leq k \leq r$, we denote by $\boldsymbol{n}_{k} \in \mathbb{Z}^{n}$ the primitive generator of $\rho_{k}$, such that

$$
\rho_{k} \cap \mathbb{Z}^{n}=\mathbb{Z}_{\geq 0} \cdot \boldsymbol{n}_{k}
$$

Note that $\rho_{k}=$ Cone $\left(\boldsymbol{n}_{k}\right)=\mathbb{R}_{\geq 0} \cdot \boldsymbol{n}_{k}$ for each $1 \leq k \leq r$.

\footnotetext{
${ }^{3}$ When $S$ is the emptyset $\emptyset$, we set $\operatorname{Cone}(\emptyset)=\left\{\mathbf{0}_{n}\right\}$ and we may also regard it as one of strongly convex rational polyhedral cones in $\mathbb{R}^{n}$, where we denote by $\mathbf{0}_{n}$ the zero vector in $\mathbb{R}^{n}$ defined by $\mathbf{0}_{n}=(0, \cdots, 0) \in \mathbb{R}^{n}$.
} 
Polyhedral products Next, recall the definition of polyhedral products.

Definition 2.2. Let $K$ be a simplicial complex on the vertex set $[r]=$ $\{1,2, \cdots, r\} \underline{4}$ and let $(\underline{X}, \underline{A})=\left\{\left(X_{1}, A_{1}\right), \cdots,\left(X_{r}, A_{r}\right)\right\}$ be a set of pairs of based spaces such that $A_{i} \subset X_{i}$ for each $1 \leq i \leq r$.

(i) Let $\mathcal{Z}_{K}(\underline{X}, \underline{A})$ denote the polyhedral product of $(\underline{X}, \underline{A})$ with respect to $K$ given by the union

$$
\begin{aligned}
\mathcal{Z}_{K}(\underline{X}, \underline{A}) & =\bigcup_{\sigma \in K}(\underline{X}, \underline{A})^{\sigma}, \quad \text { where we set } \\
(\underline{X}, \underline{A})^{\sigma} & =\left\{\left(x_{1}, \cdots, x_{r}\right) \in X_{1} \times \cdots \times X_{r}: x_{k} \in A_{k} \text { if } k \notin \sigma\right\} .
\end{aligned}
$$

When $\left(X_{i}, A_{i}\right)=(X, A)$ for each $1 \leq i \leq r$, we write $\mathcal{Z}_{K}(X, A)=\mathcal{Z}_{K}(\underline{X}, \underline{A})$.

(ii) For a subset $\sigma \subset[r]$, let $L_{\sigma}$ denote the coordinate subspace of $\mathbb{C}^{r}$ defined by

$$
L_{\sigma}=\left\{\left(x_{1}, \cdots, x_{r}\right) \in \mathbb{C}^{r}: x_{i}=0 \text { if } i \in \sigma\right\} .
$$

Define the complement $U(K)$ of the coordinate subspaces of type $K$ by

$$
U(K)=\mathbb{C}^{r} \backslash \bigcup_{\sigma \in I(K)} L_{\sigma} \quad \text { where we set } I(K)=\{\sigma \subset[r]: \sigma \notin K\}
$$

(iii) For a fan $\Sigma$ in $\mathbb{R}^{n}$, let $\mathcal{K}_{\Sigma}$ denote the underlying simplicial complex of $\Sigma$ defined by

$$
\mathcal{K}_{\Sigma}=\left\{\left\{i_{1}, \cdots, i_{s}\right\} \subset[r]: \operatorname{Cone}\left(\boldsymbol{n}_{i_{1}}, \boldsymbol{n}_{i_{2}}, \cdots, \boldsymbol{n}_{i_{s}}\right) \in \Sigma\right\} .
$$

Note that $\mathcal{K}_{\Sigma}$ is a simplicial complex on the vertex set $[r]$.

Remark 2.3. (i) It is easy to see that the following equality holds:

$$
U(K)=\mathcal{Z}_{K}\left(\mathbb{C}, \mathbb{C}^{*}\right) .
$$

(ii) The fan $\Sigma$ is completely determined by the pair $\left(\mathcal{K}_{\Sigma},\left\{\boldsymbol{n}_{k}\right\}_{k=1}^{r}\right)$.

Indeed, if we set $C(\sigma)=\operatorname{Cone}\left(\boldsymbol{n}_{i_{1}}, \cdots, \boldsymbol{n}_{i_{s}}\right)$ for $\sigma=\left\{i_{1}, \cdots, i_{s}\right\} \subset[r]$ and $C(\emptyset)=\left\{\mathbf{0}_{n}\right\}$, then it is easy to see that $\Sigma=\left\{\mathrm{C}(\sigma): \sigma \in \mathcal{K}_{\Sigma}\right\}$.

\footnotetext{
${ }^{4}$ Let $K$ be some set of subsets of $[r]$. Then the set $K$ is called an abstract simplicial complex on the vertex set $[r]$ if the following condition holds: if $\tau \subset \sigma$ and $\sigma \in K$, then $\tau \in K$. In this paper by a simplicial complex $K$ we always mean an an abstract simplicial complex, and we always assume that a simplicial complex $K$ contains the empty set $\emptyset$.
} 
Homogenous coordinates Recall the homogenous coordinates on toric varieties. Let $\Sigma$ be a fan in $\mathbb{R}^{n}$ as in Definition 2.1 .

Definition 2.4. (i) Let $G_{\Sigma} \subset \mathbb{T}_{\mathbb{C}}^{r}=\left(\mathbb{C}^{*}\right)^{r}$ denote the multiplicative subgroup of $\mathbb{T}_{\mathbb{C}}^{r}$ defined by

$$
G_{\Sigma}=\left\{\left(\mu_{1}, \cdots, \mu_{r}\right) \in \mathbb{T}_{\mathbb{C}}^{r}: \prod_{k=1}^{r}\left(\mu_{k}\right)^{\left\langle n_{k}, \boldsymbol{m}\right\rangle}=1 \text { for all } \boldsymbol{m} \in \mathbb{Z}^{n}\right\},
$$

where $\langle$,$\rangle denotes the standard inner product on \mathbb{R}^{n}$ given by $\langle\boldsymbol{u}, \boldsymbol{v}\rangle=$ $\sum_{k=1}^{n} u_{k} v_{k}$ for $\boldsymbol{u}=\left(u_{1}, \cdots, u_{n}\right)$ and $\boldsymbol{v}=\left(v_{1}, \cdots, v_{n}\right) \in \mathbb{R}^{n}$.

(ii) Consider the natural $G_{\Sigma^{-}}$action on $\mathcal{Z}_{\mathcal{K}_{\Sigma}}\left(\mathbb{C}, \mathbb{C}^{*}\right)$ given by coordinatewise multiplication, i.e.

$$
\mu \cdot \boldsymbol{x}=\left(\mu_{1} x_{1}, \cdots, \mu_{r} x_{r}\right)
$$

for $(\mu, \boldsymbol{x})=\left(\left(\mu_{1}, \cdots, \mu_{r}\right),\left(x_{1}, \cdots, x_{r}\right)\right) \in G_{\Sigma} \times \mathcal{Z}_{\mathcal{K}_{\Sigma}}\left(\mathbb{C}, \mathbb{C}^{*}\right)$. We denote by

$$
\mathcal{Z}_{\mathcal{K}_{\Sigma}}\left(\mathbb{C}, \mathbb{C}^{*}\right) / G_{\Sigma}=U\left(\mathcal{K}_{\Sigma}\right) / G_{\Sigma}
$$

the corresponding orbit space and let

$$
q_{\Sigma}: \mathcal{Z}_{\mathcal{K}_{\Sigma}}\left(\mathbb{C}, \mathbb{C}^{*}\right) \rightarrow \mathcal{Z}_{\mathcal{K}_{\Sigma}}\left(\mathbb{C}, \mathbb{C}^{*}\right) / G_{\Sigma}=U\left(\mathcal{K}_{\Sigma}\right) / G_{\Sigma}
$$

denote the canonical projection.

The following theorem, which plays a crucial role in the proof of our main result, states that in a toric variety, under certain mild conditions, one can construct "homogeneous coordinates" similar to those in a projective space.

Theorem 2.5 ([5], Theorem 2.1). If the set $\left\{\boldsymbol{n}_{k}\right\}_{k=1}^{r}$ of all primitive generators spans $\mathbb{R}^{n}$ (i.e. $\sum_{k=1}^{r} \mathbb{R} \cdot \boldsymbol{n}_{k}=\mathbb{R}^{n}$ ), there is a natural isomorphism

$$
X_{\Sigma} \cong \mathcal{Z}_{\mathcal{K}_{\Sigma}}\left(\mathbb{C}, \mathbb{C}^{*}\right) / G_{\Sigma}=U\left(\mathcal{K}_{\Sigma}\right) / G_{\Sigma}
$$

By using the above result we can obtain the following result whose proof is postponed in the last part of this section.

Lemma 2.6 (cf. [6], Theorem 3.1). Suppose that the set $\left\{\boldsymbol{n}_{k}\right\}_{k=1}^{r}$ of all primitive generators spans $\mathbb{R}^{n}$, and let $f_{k} \in \mathbb{C}\left[z_{0}, \cdots, z_{m}\right]$ be a homogenous polynomial of the degree $d_{k}^{*}$ for each $1 \leq k \leq r$ such that the polynomials $\left\{f_{k}\right\}_{k \in \sigma}$ have no common real root except $\mathbf{0}_{m+1} \in \mathbb{R}^{m+1}$ for each $\sigma \in I\left(\mathcal{K}_{\Sigma}\right)$. Then there is a unique map $f: \mathbb{R P}^{m} \rightarrow X_{\Sigma}$ such that the following diagram

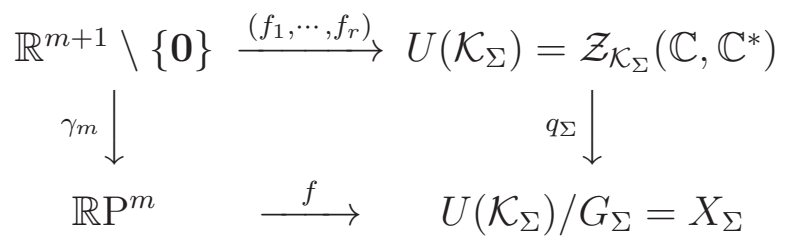


is commutative if and only if $\sum_{k=1}^{r} d_{k}^{*} \boldsymbol{n}_{k}=\mathbf{0}_{n}$, where $\gamma_{m}: \mathbb{R}^{m+1} \backslash\{\mathbf{0}\} \rightarrow$ $\mathbb{R P}^{m}$ denotes the canonical double covering and the map $q_{\Sigma}$ is the canonical projection induced from the identification (2.14).

Remark 2.7. We call the map $f$ determined by an $r$-tuple $\left(f_{1}, \cdots, f_{r}\right)$ of homogenous polynomials as an algebraic map and we write $f=\left[f_{1}, \cdots, f_{r}\right]$. Note that two different such $r$-tuples of polynomials can determine the same maps. For example, suppose that $\left(f_{1}, \cdots, f_{r}\right)$ is the $r$-tuple of homogenous polynomials in $\mathbb{C}\left[z_{0}, \cdots, z_{m}\right]$ of degree $d_{1}^{*}, \cdots, d_{r}^{*}$ satisfying the condition given in Lemma 2.6. Then if $\left(a_{1}, \cdots, a_{r}\right) \in \mathbb{N}^{r}$ is the $r$-tuple of positive integers and it satisfies the condition $\sum_{k=1}^{r} d_{k}^{*} \boldsymbol{n}_{k}=\sum_{k=1}^{r} a_{k} \boldsymbol{n}_{k}=\mathbf{0}_{n}$, we can easily see that $f=\left[f_{1}, \cdots, f_{r}\right]=\left[g^{a_{1}} f_{1}, \cdots, g^{a_{r}} f_{r}\right]=\left[h^{a_{1}} f_{1}, \cdots, h^{a_{r}} f_{r}\right]$ for $g=\sum_{k=0}^{m} z_{k}^{2}$ and $h=\left(z_{0}+z_{1}\right)^{2}+\sum_{k=2}^{m} z_{k}^{2}$.

Assumptions Let $\Sigma$ be a fan in $\mathbb{R}^{n}$ satisfying the condition (2.3) as in Definition 2.1. From now on, we assume that the following two conditions hold.

(2.14.1) There is an $r$-tuple $D_{*}=\left(d_{1}^{*}, \cdots, d_{r}^{*}\right) \in \mathbb{N}^{r}$ of positive integers such that $\sum_{k=1}^{r} d_{k}^{*} \boldsymbol{n}_{k}=\mathbf{0}_{n}$.

(2.14,2) The set $\left\{\boldsymbol{n}_{k}\right\}_{k=1}^{r}$ of primitive generators spans $\mathbb{Z}^{n}$ over $\mathbb{Z}$.

Remark 2.8. (i) Note that $X_{\Sigma}$ is a compact iff $\bigcup_{\sigma \in \Sigma} \sigma=\mathbb{R}^{n}[7$, Theorem 3.4.1]. Note also that $X_{\Sigma}$ is simply connected if and only if $\sum_{k=1}^{r} \mathbb{Z} \cdot \boldsymbol{n}_{k}=\mathbb{Z}^{n}$ (see Lemma 3.4 below). Hence, the condition (2.14,2) always holds if $X_{\Sigma}$ is compact or simply connected. On the other hand, if the condition (2.14,2) holds, one can easily see that the set $\left\{\boldsymbol{n}_{k}\right\}_{k=1}^{r}$ spans $\mathbb{R}^{n}$ over $\mathbb{R}$, and there is an isomorphism (2.14) for the space $X_{\Sigma}$.

(ii) We know that the condition (2.14,1) holds if $X_{\Sigma}$ is compact and nonsingular [6, Theorem 3.1].

(iii) Let $\Sigma$ denote the fan in $\mathbb{R}^{2}$ given by $\Sigma=\left\{\left\{\boldsymbol{0}_{2}\right\}\right.$, Cone $\left(\boldsymbol{n}_{1}\right)$, Cone $\left.\left(\boldsymbol{n}_{2}\right)\right\}$ for the standard basis $\boldsymbol{n}_{1}=\boldsymbol{e}_{1}=(1,0), \boldsymbol{n}_{2}=\boldsymbol{e}_{2}=(0,1)$. Then the toric variety $X_{\Sigma}$ of $\Sigma$ is $\mathbb{C}^{2}$ which has trivial homogenous coordinates. It is clearly a (simply connected) smooth toric variety, and the condition (2.14.1) also holds. However, in this case, $\sum_{k=1}^{2} d_{k}^{*} \boldsymbol{n}_{k}=\mathbf{0}_{2}$ iff $\left(d_{1}^{*}, d_{2}^{*}\right)=(0,0)$. Hence, it follows from Lemma 2.6 that there are no algebraic maps $\mathbb{R P}^{m} \rightarrow X_{\Sigma}=\mathbb{C}^{2}$ other than the constant maps. Assuming the condition (2.14.1) guarantees the existence of non-trivial algebraic maps $\mathbb{R P}^{m} \rightarrow X_{\Sigma}$. Of course, it would be sufficient to assume that $D=\left(d_{1}, \ldots, d_{r}\right) \neq(0, \ldots 0)$ but if $d_{i}=0$ for some $i$, then the number $d(D, \Sigma)$ (defined in (2.25)) is not a positive integer and our assertion (Theorem 2.16 below) is vacuous. For this reason, we will assume the condition $d_{k}^{*} \geq 1$ for each $1 \leq k \leq r$ in (2.14.1). 
Spaces of tuples of polynomials which define algebraic maps Let $X_{\Sigma}$ be a non-singular toric variety and make the identification $X_{\Sigma}=U\left(\mathcal{K}_{\Sigma}\right) / G_{\Sigma}$. Let $z_{0}, \cdots, z_{m}$ be variables.

Now we consider the space of all tuples of polynomials which define based algebraic maps.

Definition 2.9. (i) For each $d, m \in \mathbb{N}$, let $\mathcal{H}_{m}^{d}(\mathbb{C})$ denote the space of all homogenous polynomials $f\left(z_{0}, \cdots, z_{m}\right) \in \mathbb{C}\left[z_{0}, \cdots, z_{m}\right]$ of degree $d$.

(ii) For each $r$-tuple $D=\left(d_{1}, \cdots, d_{r}\right) \in \mathbb{N}^{r}$, let $\operatorname{Pol}_{D}^{*}\left(\mathbb{R P} P^{m}, X_{\Sigma}\right)$ denote the space of $r$-tuples

$$
f=\left(f_{1}\left(z_{0}, \cdots, z_{m}\right), \cdots, f_{r}\left(z_{0}, \cdots, z_{m}\right)\right) \in \mathcal{H}_{m}^{d_{1}}(\mathbb{C}) \times \cdots \times \mathcal{H}_{m}^{d_{r}}(\mathbb{C})
$$

of homogenous polynomials satisfying the following two conditions:

(2.15.1) $f(\boldsymbol{x})=\left(f_{1}(\boldsymbol{x}), \cdots, f_{r}(\boldsymbol{x})\right) \in U\left(\mathcal{K}_{\Sigma}\right)$ for any point $\boldsymbol{x}=\left(x_{0}, \cdots, x_{m}\right) \in$ $\mathbb{R}^{m+1} \backslash\left\{\mathbf{0}_{m+1}\right\}$.

(2.15.2) $f\left(\boldsymbol{e}_{1}\right)=\left(f_{1}\left(\boldsymbol{e}_{1}\right), \cdots, f_{r}\left(\boldsymbol{e}_{1}\right)\right)=(1,1, \cdots, 1)$, i.e. the coefficient of $\left(z_{0}\right)^{d_{k}}$ in $f_{k}\left(z_{0}, \cdots, z_{m}\right)$ is 1 for each $1 \leq k \leq r$, where we write $\boldsymbol{e}_{1}=(1,0, \cdots, 0) \in \mathbb{R}^{m+1}$

Definition 2.10. We always assume the identification $X_{\Sigma}=U\left(\mathcal{K}_{\Sigma}\right) / G_{\Sigma}$, denote by $\left[y_{1}, \cdots, y_{r}\right]$ the point in $X_{\Sigma}$ represented by $\left(y_{1}, \cdots, y_{r}\right) \in U\left(\mathcal{K}_{\Sigma}\right)$, and choose the two points $[1: 0: \cdots: 0] \in \mathbb{R P}^{m}$ and $*=[1, \cdots, 1] \in X_{\Sigma}$ as the base-points of $\mathbb{R P}^{m}$ and $X_{\Sigma}$ respectively.

Let $D=\left(d_{1}, \cdots, d_{r}\right) \in \mathbb{N}^{r}$ be an $r$-tuple of positive integers such that $\sum_{k=1}^{r} d_{k} \boldsymbol{n}_{k}=\mathbf{0}_{n}$. Then by using Lemma 2.6, for each $r$-tuple

$$
f=\left(f_{1}\left(z_{0}, \cdots, z_{m}\right), \cdots, f_{r}\left(z_{0}, \cdots, z_{m}\right)\right) \in \operatorname{Pol}_{D}^{*}\left(\mathbb{R P}^{m}, X_{\Sigma}\right)
$$

one can define based algebraic map

$$
\begin{gathered}
{[f]=\left[f_{1}, \cdots, f_{r}\right]:\left(\mathbb{R P}^{m},\left[\boldsymbol{e}_{1}\right]\right) \rightarrow\left(X_{\Sigma}, *\right) \text { by }} \\
{[f]([\boldsymbol{x}])=\left[f_{1}(\boldsymbol{x}), \cdots, f_{r}(\boldsymbol{x})\right]}
\end{gathered}
$$

for $[\boldsymbol{x}]=\left[x_{0}: \cdots: x_{m}\right] \in \mathbb{R} \mathrm{P}^{m}$, where $\boldsymbol{x}=\left(x_{0}, \cdots, x_{m}\right) \in \mathbb{R}^{m+1} \backslash\left\{\mathbf{0}_{m+1}\right\}$. Hence, we obtain the natural map

$$
\begin{gathered}
i_{D, m}: \operatorname{Pol}_{D}^{*}\left(\mathbb{R P}^{m}, X_{\Sigma}\right) \rightarrow \operatorname{Map}_{D}^{*}\left(\mathbb{R P}^{m}, X_{\Sigma}\right) \text { given by } \\
i_{D, m}(f)=[f]=\left[f_{1}, \cdots, f_{r}\right]
\end{gathered}
$$

for $f=\left(f_{1}\left(z_{0}, \cdots, z_{m}\right), \cdots, f_{r}\left(z_{0}, \cdots, z_{m}\right)\right) \in \operatorname{Pol}_{D}^{*}\left(\mathbb{R P}^{m}, X_{\Sigma}\right)$, where we denote by $\operatorname{Map}_{D}^{*}\left(\mathbb{R P}^{m}, X_{\Sigma}\right)$ the path-component of $\operatorname{Map}^{*}\left(\mathbb{R P}^{m}, X_{\Sigma}\right)$ which contains all algebraic maps of degree $D$. 
Remark 2.11. When $m=1$, we make the identification $\mathbb{R} P^{1}=S^{1}=\mathbb{R} \cup \infty$ and choose the points $\infty$ as the base-point of $\mathbb{R P}^{1}$. Then, by setting $z=\frac{z_{0}}{z_{1}}$, we can view a homogenous polynomial $f\left(z_{0}, z_{1}\right) \in \mathbb{C}\left[z_{0}, z_{1}\right]$ of degree $d$ as a monic polynomial $f_{k}(z) \in \mathbb{C}[z]$ of degree $d$.

Thus, when $m=1$, one can redefine the space $\operatorname{Pol}_{D}^{*}\left(S^{1}, X_{\Sigma}\right)$ as follows.

Definition 2.12. (i) Let $\mathrm{P}^{d}(\mathbb{C})$ denote the space of all monic polynomials $f(z)=z^{d}+a_{1} z^{d-1}+\cdots+a_{d-1} z+a_{d} \in \mathbb{C}[z]$ of degree $d$, and let

$$
\mathrm{P}^{D}=\mathrm{P}^{d_{1}}(\mathbb{C}) \times \mathrm{P}^{d_{2}}(\mathbb{C}) \times \cdots \times \mathrm{P}^{d_{r}}(\mathbb{C}) .
$$

Note that there is a homeomorphism $\phi: \mathrm{P}^{d}(\mathbb{C}) \cong \mathbb{C}^{d}$ given by $\phi\left(z^{d}+\right.$ $\left.\sum_{k=1}^{d} a_{k} z^{d-k}\right)=\left(a_{1}, \cdots, a_{d}\right) \in \mathbb{C}^{d}$.

(ii) For any $r$-tuple $D=\left(d_{1}, \cdots, d_{r}\right) \in \mathbb{N}^{r}$, let $\operatorname{Pol}_{D}^{*}\left(S^{1}, X_{\Sigma}\right)$ denote the space of all $r$-tuples $\left(f_{1}(z), \cdots, f_{r}(z)\right) \in \mathrm{P}^{D}$ of monic polynomials satisfying the following condition $(\dagger)$ :

$(\dagger)$ The polynomials $f_{i_{1}}(z), \cdots, f_{i_{s}}(z)$ have no common real root for any $\sigma=\left\{i_{1}, \cdots, i_{s}\right\} \in I\left(\mathcal{K}_{\Sigma}\right)$, i.e. $\left(f_{i_{1}}(\alpha), \cdots, f_{i_{s}}(\alpha)\right) \neq \mathbf{0}_{s}$ for any $\alpha \in \mathbb{R}$.

When the condition $\sum_{k=1}^{r} d_{k} \boldsymbol{n}_{k}=\mathbf{0}_{n}$ holds, by identifying $X_{\Sigma}=U\left(\mathcal{K}_{\Sigma}\right) / G_{\Sigma}$ and $\mathbb{R P}^{1}=S^{1}=\mathbb{R} \cup \infty$, one can define a natural map

$$
\begin{gathered}
i_{D}=i_{D, 1}: \operatorname{Pol}_{D}^{*}\left(S^{1}, X_{\Sigma}\right) \rightarrow \operatorname{Map}^{*}\left(S^{1}, X_{\Sigma}\right)=\Omega X_{\Sigma} \quad \text { by } \\
i_{D}\left(f_{1}(z), \cdots, f_{r}(z)\right)(\alpha)= \begin{cases}{\left[f_{1}(\alpha), \cdots, f_{r}(\alpha)\right]} & \text { if } \alpha \in \mathbb{R} \\
{[1,1, \cdots, 1]} & \text { if } \alpha=\infty\end{cases}
\end{gathered}
$$

for $\left(f_{1}(z), \cdots, f_{r}(z)\right) \in \operatorname{Pol}_{D}^{*}\left(S^{1}, X_{\Sigma}\right)$ and $\alpha \in S^{1}=\mathbb{R} \cup \infty$, where we choose the points $\infty$ and $[1,1, \cdots, 1]$ as the base-points of $S^{1}$ and $X_{\Sigma}$.

Note that $\operatorname{Pol}_{D}^{*}\left(S^{1}, X_{\Sigma}\right)$ is simply connected (which will be proved in Proposition 5.9) and that the map $\Omega q_{\Sigma}: \Omega U\left(\mathcal{K}_{\Sigma}\right) \rightarrow \Omega X_{\Sigma}$ is a universal covering (which will be shown in Lemma 3.4). Thus, when $\sum_{k=1}^{r} d_{k} \boldsymbol{n}_{k}=\mathbf{0}_{n}$, the map $i_{D}$ lifts to the space $\Omega \mathcal{Z}_{\mathcal{K}_{\Sigma}}$ and there is a map

$$
j_{D}: \operatorname{Pol}_{D}^{*}\left(S^{1}, X_{\Sigma}\right) \rightarrow \Omega U\left(\mathcal{K}_{\Sigma}\right) \simeq \Omega \mathcal{Z}_{\mathcal{K}_{\Sigma}}
$$

such that

$$
\Omega q_{\Sigma} \circ j_{D}=i_{D}
$$


Remark 2.13. (i) Note that $\operatorname{Pol}_{D}^{*}\left(S^{1}, X_{\Sigma}\right)$ is path-connected (see (ii) of Remark 7.4), and that $X_{\Sigma}$ is simply connected if the condition (2.14.2) is satisfied (see (i) of Lemma 3.4).

(ii) Even if $\sum_{k=1}^{r} d_{k} \boldsymbol{n}_{k} \neq \mathbf{0}_{n}$ we can define the two maps

$$
i_{D}: \operatorname{Pol}_{D}^{*}\left(S^{1}, X_{\Sigma}\right) \rightarrow \Omega X_{\Sigma}, \quad j_{D}: \operatorname{Pol}_{D}^{*}\left(S^{1}, X_{\Sigma}\right) \rightarrow \Omega U\left(\mathcal{K}_{\Sigma}\right)
$$

and this will be done in 96 (see (6.17) in detail).

Now we need to define the numbers $r_{\min }(\Sigma)$ and $d(D, \Sigma)$.

Definition 2.14. Let $\Sigma$ be a fan in $\mathbb{R}^{n}$ as in Definition 2.1

(i) We say that a set $S=\left\{\boldsymbol{n}_{i_{1}}, \cdots, \boldsymbol{n}_{i_{s}}\right\}$ is primitive in $\Sigma$ if Cone $(S) \notin \Sigma$ but $\operatorname{Cone}(T) \in \Sigma$ for any proper subset $T \varsubsetneqq S$.

(ii) For $D=\left(d_{1}, \cdots, d_{r}\right) \in \mathbb{N}^{r}$ define integers $r_{\min }(\Sigma)$ and $d(D, \Sigma ; m)$ by

$$
\left\{\begin{array}{cl}
r_{\min }(\Sigma) & =\min \left\{s \in \mathbb{N}:\left\{\boldsymbol{n}_{i_{1}}, \cdots, \boldsymbol{n}_{i_{s}}\right\} \text { is primitive in } \Sigma\right\} \\
d(D, \Sigma ; m) & =\left(2 r_{\min }(\Sigma)-m-1\right) d_{\min }-2, \text { where } \\
d_{\text {min }} & =\min \left\{d_{1}, \cdots, d_{r}\right\} .
\end{array}\right.
$$

In particular, when $m=1$ we also define the positive integer $d(D, \Sigma)$ by

$$
d(D, \Sigma)=d(D, \Sigma ; 1)=\left(2 r_{\min }(\Sigma)-2\right) d_{\min }-2 .
$$

Now recall the following result.

Theorem 2.15 (14]). Let $m \geq 2$ be a positive integer, $X_{\Sigma}$ be a compact smooth toric variety and $D=\left(d_{1}, \cdots, d_{r}\right) \in \mathbb{N}^{r}$ be an $r$-tuple of positive integers such that $\sum_{k=1}^{r} d_{k} \boldsymbol{n}_{k}=\mathbf{0}_{n}$. Then the natural map

$$
i_{D, m}: \operatorname{Pol}_{D}^{*}\left(\mathbb{R P}^{m}, X_{\Sigma}\right) \rightarrow \operatorname{Map}_{D}^{*}\left(\mathbb{R P}^{m}, X_{\Sigma}\right)
$$

is a homology equivalence through dimension $d(D, \Sigma ; m)$.

Note that the above result does not hold for the case $m=1$. For example, this can be seen in [13] for the case $X_{\Sigma}=\mathbb{C P}^{n}$. In fact, the main purpose of this paper is to investigate the result corresponding to this theorem for the case $m=1$.

The main results More precisely the main results of this paper are as follows. 
Theorem 2.16. Let $D=\left(d_{1}, \cdots, d_{r}\right) \in \mathbb{N}^{r}$ be an $r$-tuple of positive integers satisfying the condition $\sum_{k=1}^{r} d_{k} \boldsymbol{n}_{k}=\mathbf{0}_{n}$, and let $X_{\Sigma}$ be a simply connected non-singular toric variety such that the condition (2.14.1) holds.

Then the map

$$
j_{D}: \operatorname{Pol}_{D}^{*}\left(S^{1}, X_{\Sigma}\right) \rightarrow \Omega U\left(\mathcal{K}_{\Sigma}\right) \simeq \Omega \mathcal{Z}_{\mathcal{K}_{\Sigma}}
$$

is a homotopy equivalence through dimension $d(D, \Sigma)$.

Corollary 2.17. Under the same assumption as in Theorem 2.16, the map $i_{D}: \operatorname{Pol}_{D}^{*}\left(S^{1}, X_{\Sigma}\right) \rightarrow \Omega X_{\Sigma}$ induces an isomorphism

$$
\left(i_{D}\right)_{*}: \pi_{k}\left(\mathrm{Pol}_{D}^{*}\left(S^{1}, X_{\Sigma}\right)\right) \stackrel{\cong}{\longrightarrow} \pi_{k}\left(\Omega X_{\Sigma}\right) \cong \pi_{k+1}\left(X_{\Sigma}\right)
$$

for any $2 \leq k \leq d(D, \Sigma)$.

Corollary 2.18. Let $D=\left(d_{1}, \cdots, d_{r}\right) \in \mathbb{N}^{r}$ be an $r$-tuple of positive integers satisfying the condition $\sum_{k=1}^{r} d_{k} \boldsymbol{n}_{k}=\mathbf{0}_{n}$, and let $X_{\Sigma}$ be a simply connected compact non-singular toric variety. Let $\Sigma(1)$ denote the set of all one dimensional cones in $\Sigma$, and $\Sigma_{1}$ any fan in $\mathbb{R}^{n}$ such that $\Sigma(1) \subset \Sigma_{1} \varsubsetneqq \Sigma$.

(i) Then $X_{\Sigma_{1}}$ is a non-singular open toric subvariety of $X_{\Sigma}$ and the map

$$
j_{D}: \operatorname{Pol}_{D}^{*}\left(S^{1}, X_{\Sigma_{1}}\right) \rightarrow \Omega U\left(\mathcal{K}_{\Sigma}\right) \simeq \Omega \mathcal{Z}_{\Sigma_{1}}
$$

is a homotopy equivalence through dimension $d\left(D, \Sigma_{1}\right)$.

(ii) Moreover, the map $i_{D}: \operatorname{Pol}_{D}^{*}\left(S^{1}, X_{\Sigma_{1}}\right) \rightarrow \Omega X_{\Sigma_{1}}$ induces the isomorphism

$$
\left(i_{D}\right)_{*}: \pi_{k}\left(\operatorname{Pol}_{D}^{*}\left(S^{1}, X_{\Sigma_{1}}\right)\right) \stackrel{\cong}{\longrightarrow} \pi_{k}\left(\Omega X_{\Sigma_{1}}\right) \cong \pi_{k+1}\left(X_{\Sigma_{1}}\right)
$$

for any $2 \leq k \leq d\left(D, \Sigma_{1}\right)$.

Remark 2.19. If $X_{\Sigma}$ is compact, the condition (2.14,1) is satisfied (see (ii) of Remark 2.8) and one can prove the above two results (Theorem 2.16, Corollary 2.17) by using [14, Theorem 6.2]. However, the proof given in [14] uses the Stone-Weierstrass theorem and it cannot be applied when $X_{\Sigma}$ is not compact.

Finally consider the $r$-tuple $D=\left(d_{1}, \cdots, d_{r}\right) \in \mathbb{N}^{r}$ such that $\sum_{k=1}^{r} d_{k} \boldsymbol{n}_{k} \neq$ $\mathbf{0}_{n}$. Then the map $i_{D}$ is not well-defined (see Lemma 2.6). However, even in this situation we have a map $j_{D}: \operatorname{Pol}_{D}^{*}\left(S^{1}, X_{\Sigma}\right) \rightarrow \Omega \mathcal{Z}_{\mathcal{K}_{\Sigma}}$ and the following result holds. 
Theorem 2.20. Let $D=\left(d_{1}, \cdots, d_{r}\right) \in \mathbb{N}^{r}$ be an $r$-tuple of positive integers such that $\sum_{k=1}^{r} d_{k} \boldsymbol{n}_{k} \neq \mathbf{0}_{n}$, and let $X_{\Sigma}$ be a simply connected non-singular toric variety such that the condition 2.14.1) holds.

Then the map defined by (6.16)

$$
j_{D}: \operatorname{Pol}_{D}^{*}\left(S^{1}, X_{\Sigma}\right) \rightarrow \Omega U\left(\mathcal{K}_{\Sigma}\right) \simeq \Omega \mathcal{Z}_{\mathcal{K}_{\Sigma}}
$$

is a homotopy equivalence thorough dimension $d(D, \Sigma)$.

Corollary 2.21. Under the same assumption as Theorem 2.20, the map

$$
i_{D}=\left(\Omega q_{\Sigma}\right) \circ j_{D}: \operatorname{Pol}_{D}^{*}\left(S^{1}, X_{\Sigma}\right) \rightarrow \Omega X_{\Sigma}
$$

induces an isomorphism

$$
\left(i_{D}\right)_{*}: \pi_{k}\left(\mathrm{Pol}_{D}^{*}\left(S^{1}, X_{\Sigma}\right)\right) \stackrel{\cong}{\longrightarrow} \pi_{k}\left(\Omega X_{\Sigma}\right) \cong \pi_{k+1}\left(X_{\Sigma}\right)
$$

for any $2 \leq k \leq d(D, \Sigma)$.

Since $X_{\Sigma}$ is compact and $\Sigma(1) \subset \Sigma_{1} \varsubsetneqq \Sigma$, the condition (2.14,1) holds for the fan $\Sigma_{1}$ and we obtain the following result by using Theorem 2.20 and Corollary 2.21.

Corollary 2.22. Let $D=\left(d_{1}, \cdots, d_{r}\right) \in \mathbb{N}^{r}$ be an $r$-tuple of positive integers, and let $X_{\Sigma}$ be a simply connected compact non-singular toric variety. Let $\Sigma(1)$ denote the set of all one dimensional cones in $\Sigma$, and $\Sigma_{1}$ any fan in $\mathbb{R}^{n}$ such that $\Sigma(1) \subset \Sigma_{1} \varsubsetneqq \Sigma$.

(i) Then $X_{\Sigma_{1}}$ is a non-singular open toric subvariety of $X_{\Sigma}$ and the map

$$
j_{D}: \operatorname{Pol}_{D}^{*}\left(S^{1}, X_{\Sigma_{1}}\right) \rightarrow \Omega U\left(\mathcal{K}_{\Sigma_{1}}\right) \simeq \Omega \mathcal{Z}_{\Sigma_{1}}
$$

is a homotopy equivalence through dimesnion $d\left(D, \Sigma_{1}\right)$.

(ii) Furthermore, the map $i_{D}: \operatorname{Pol}_{D}^{*}\left(S^{1}, X_{\Sigma_{1}}\right) \rightarrow \Omega X_{\Sigma_{1}}$ induces the isomorphism

$$
\left(i_{D}\right)_{*}: \pi_{k}\left(\operatorname{Pol}_{D}^{*}\left(S^{1}, X_{\Sigma_{1}}\right)\right) \stackrel{\cong}{\longrightarrow} \pi_{k}\left(\Omega X_{\Sigma_{1}}\right) \cong \pi_{k+1}\left(X_{\Sigma_{1}}\right)
$$

for any $2 \leq k \leq d\left(D, \Sigma_{1}\right)$.

Remark 2.23. The map $i_{D}$ is well-defined only when $\sum_{k=1}^{r} d_{k} \boldsymbol{n}_{k}=\mathbf{0}_{n}$. Hence, when $\sum_{k=1}^{r} d_{k} \boldsymbol{n}_{k} \neq \mathbf{0}_{n}$, there is no map $j_{D}: \operatorname{Pol}_{D}^{*}\left(S^{1}, X_{\Sigma}\right) \rightarrow \Omega \mathcal{Z}_{\mathcal{K}_{\Sigma}}$ satisfying the condition (2.23). However, even if $\sum_{k=1}^{r} d_{k} \boldsymbol{n}_{k} \neq \mathbf{0}_{n}$ we can define the map $j_{D}: \operatorname{Pol}_{D}^{*}\left(S^{1}, X_{\Sigma}\right) \rightarrow \Omega \mathcal{Z}_{\mathcal{K}_{\Sigma}}$ by using the map $j_{D_{0}}$ (for some $\left.D_{0}=\left(m_{0} d_{1}^{*}, \cdots, m_{0} d_{r}^{*}\right) \in \mathbb{N}^{r}\right)$ and the stabilization map $s_{D, D_{0}}$. In fact, In this case the maps $j_{D}$ and $i_{D}$ are defined by $j_{D}=j_{D_{0}} \circ s_{D, D_{0}}$ and $i_{D}=\Omega q_{\Sigma} \circ j_{D}$ (see (6.16) and (9.1) in detail). So the condition (2.23) still holds. 
Examples Since the case $X_{\Sigma}=\mathbb{C P}^{n}$ of Corollary 2.18 was treated in [15], consider the case that $X_{\Sigma}$ is the Hirzerbruch surface $H(k)$.

Definition 2.24. For an integer $k \in \mathbb{Z}$, let $H(k)$ be the Hirzerbruch surface defined by

$$
H(k)=\left\{\left(\left[x_{0}: x_{1}: x_{2}\right],\left[y_{1}: y_{2}\right]\right) \in \mathbb{C P}^{2} \times \mathbb{C P}^{1}: x_{1} y_{1}^{k}=x_{2} y_{2}^{k}\right\} \subset \mathbb{C P}^{2} \times \mathbb{C P}^{1} .
$$

Since there are isomorphisms $H(-k) \cong H(k)$ for $k \neq 0$ and $H(0) \cong \mathbb{C P}^{1} \times$ $\mathbb{C P}^{1}$, without loss of generality we can assume that $k \geq 1$. Let $\Sigma_{k}$ denote the fan in $\mathbb{R}^{2}$ given by

$\Sigma_{k}=\left\{\operatorname{Cone}\left(\mathbf{n}_{i}, \mathbf{n}_{i+1}\right)(1 \leq i \leq 3), \operatorname{Cone}\left(\mathbf{n}_{4}, \mathbf{n}_{1}\right), \operatorname{Cone}\left(\boldsymbol{n}_{j}\right)(1 \leq j \leq 4),\{\mathbf{0}\}\right\}$,

where we set $\boldsymbol{n}_{1}=(1,0), \boldsymbol{n}_{2}=(0,1), \boldsymbol{n}_{3}=(-1, k), \boldsymbol{n}_{4}=(0,-1)$.

It is easy to see that $\Sigma_{k}$ is the fan of $H(k)$ and that $H(k)$ is a compact non-singular toric variety. Note that $\Sigma_{k}(1)=\left\{\operatorname{Cone}\left(\boldsymbol{n}_{i}\right): 1 \leq i \leq 4\right\}$. Since $\left\{\boldsymbol{n}_{1}, \boldsymbol{n}_{3}\right\}$ and $\left\{\boldsymbol{n}_{2}, \boldsymbol{n}_{4}\right\}$ are only primitive in $\Sigma_{k}, r_{\min }\left(\Sigma_{k}\right)=2$.

Moreover, for $D=\left(d_{1}, d_{2}, d_{3}, d_{4}\right) \in \mathbb{N}^{4}$ the equality $\sum_{k=1}^{4} d_{k} \boldsymbol{n}_{k}=\mathbf{0}_{2}$ holds iff $\left(d_{3}, d_{4}\right)=\left(d_{1}, k d_{1}+d_{2}\right)$. Thus, if $\sum_{k=1}^{4} d_{k} \boldsymbol{n}_{k}=\mathbf{0}_{2}$, we have $d_{\min }=$ $\min \left\{d_{1}, d_{2}, d_{3}, d_{4}\right\}=\min \left\{d_{1}, d_{2}\right\}$.

By Corollary 2.18 and Corollary 2.22 we have:

Example 2.25. Let $D=\left(d_{1}, d_{2}, d_{3}, d_{4}\right) \in \mathbb{N}^{4}, k \in \mathbb{N}$, and $\Sigma$ be a fan in $\mathbb{R}^{2}$ such that $\Sigma_{k}(1)=\left\{\right.$ Cone $\left.\left(\boldsymbol{n}_{i}\right): 1 \leq i \leq 4\right\} \subset \Sigma \subset \Sigma_{k}$ as in Definition 2.24.

(i) $X_{\Sigma}$ is a non-singular open toric subvariety of $H(k)$ if $\Sigma \varsubsetneqq \Sigma_{k}$.

(ii) If $\sum_{k=1}^{4} d_{k} \boldsymbol{n}_{k}=\mathbf{0}_{2}$, the equality $\left(d_{3}, d_{4}\right)=\left(d_{1}, k d_{1}+d_{2}\right)$ holds and the map $j_{D}: \operatorname{Pol}_{D}^{*}\left(S^{1}, X_{\Sigma}\right) \rightarrow \Omega \mathcal{Z}_{\mathcal{K}_{\Sigma}}$ is a homotopy equivalence through dimension $2 \min \left\{d_{1}, d_{2}\right\}-2$. Moreover, the map $i_{D}: \operatorname{Pol}_{D}^{*}\left(S^{1}, X_{\Sigma}\right) \rightarrow \Omega X_{\Sigma}$ induces an isomorphism

$$
\left(i_{D}\right)_{*}: \pi_{k}\left(\mathrm{Pol}_{D}^{*}\left(S^{1}, X_{\Sigma}\right)\right) \stackrel{\cong}{\longrightarrow} \pi_{k}\left(\Omega X_{\Sigma}\right) \cong \pi_{k+1}\left(X_{\Sigma}\right)
$$

for any $2 \leq k \leq 2 \min \left\{d_{1}, d_{2}\right\}-2$.

(iii) If $\sum_{k=1}^{4} d_{k} \boldsymbol{n}_{k} \neq \mathbf{0}_{2}$, the map $j_{D}: \operatorname{Pol}_{D}^{*}\left(S^{1}, X_{\Sigma}\right) \rightarrow \Omega \mathcal{Z}_{\mathcal{K}_{\Sigma}}$ is a homotopy equivalence through dimension $2 \min \left\{d_{1}, d_{2}, d_{3}, d_{4}\right\}-2$, and the map $i_{D}: \operatorname{Pol}_{D}^{*}\left(S^{1}, X_{\Sigma}\right) \rightarrow \Omega X_{\Sigma}$ induces an isomorphism

$$
\left(i_{D}\right)_{*}: \pi_{k}\left(\mathrm{Pol}_{D}^{*}\left(S^{1}, X_{\Sigma}\right)\right) \stackrel{\cong}{\longrightarrow} \pi_{k}\left(\Omega X_{\Sigma}\right) \cong \pi_{k+1}\left(X_{\Sigma}\right)
$$

for any $2 \leq k \leq 2 \min \left\{d_{1}, d_{2}, d_{3}, d_{4}\right\}-2$. 
Remark 2.26. (i) There are 15 non isomorphic (as varieties) non-compact subvarieties $X_{\Sigma}$ of $H(k)$ which satisfy the assumption of Corollary 2.25.

(ii) Note that there is an isomorphism

$$
\pi_{2}\left(X_{\Sigma}\right) \cong \mathbb{Z}^{r-n} \quad \text { (see Lemma } 3.4 \text { below) }
$$

in general. So $(r-n)$ of the $r$ positive integers $\left\{d_{k}\right\}_{k=1}^{r}$ can be chosen freely. For example, in Example 2.24, $(r, n)=(4,2)$ and $r-n=4-2=2$. In this case, only two positive integers $d_{1}, d_{2}$ can be chosen freely and the other integers $d_{3}$ and $d_{4}$ are determined by uniquely as $\left(d_{3}, d_{4}\right)=\left(d_{1}, k d_{1}+d_{2}\right)$.

Finally in this section we give the proof of Lemma 2.6.

Proof of Lemma 2.6. It follows from the assumptions that we can identify $X_{\Sigma}=U\left(\mathcal{K}_{\Sigma}\right) / G_{\Sigma}$ and that the map $F=\left(f_{1}, \cdots, f_{r}\right): \mathbb{R}^{m+1} \backslash\left\{\mathbf{0}_{m+1}\right\} \rightarrow$ $U\left(\mathcal{K}_{\Sigma}\right)$ is well-defined. Thus, it suffices to show that $F(\lambda \boldsymbol{x})=F(\boldsymbol{x})$ up to $G_{\Sigma^{-}}$action for any $(\lambda, \boldsymbol{x}) \in \mathbb{R}^{*} \times\left(\mathbb{R}^{m+1} \backslash\left\{\mathbf{0}_{m+1}\right\}\right)$ iff $\sum_{k=1}^{r} d_{k} \boldsymbol{n}_{k}=\mathbf{0}_{n}$. Since $f_{k}$ is a homogenous polynomial of degree $d_{k}$, by using (2.11) we have

$$
\begin{aligned}
F(\lambda \boldsymbol{x}) & =\left(f_{1}(\lambda \boldsymbol{x}), \cdots, f_{r}(\lambda \boldsymbol{x})\right)=\left(\lambda^{d_{1}} f_{1}(\boldsymbol{x}), \cdots, \lambda^{d_{r}} f_{r}(\boldsymbol{x})\right) \\
& =\left(\lambda^{d_{1}}, \cdots, \lambda^{d_{r}}\right) \cdot\left(f_{1}(\boldsymbol{x}), \cdots, f_{r}(\boldsymbol{x})\right) .
\end{aligned}
$$

Hence, it remains to show that $\left(\lambda^{d_{1}}, \cdots, \lambda^{d_{r}}\right) \in G_{\Sigma}$ for any $\lambda \in \mathbb{R}^{*}$ iff $\sum_{k=1}^{r} d_{k} \boldsymbol{n}_{k}=\mathbf{0}_{n}$. However, $\left(\lambda^{d_{1}}, \cdots, \lambda^{d_{r}}\right) \in G_{\Sigma}$ for any $\lambda \in \mathbb{R}^{*}$ iff

$$
\prod_{k=1}^{r}\left(\lambda^{d_{k}}\right)^{\left\langle\boldsymbol{n}_{k}, \boldsymbol{m}\right\rangle}=\lambda^{\left\langle\sum_{k=1}^{r} d_{k} \boldsymbol{n}_{k}, \boldsymbol{m}\right\rangle}=1 \text { for any } \boldsymbol{m} \in \mathbb{Z}^{n} \Leftrightarrow \sum_{k=1}^{r} d_{k} \boldsymbol{n}_{k}=\mathbf{0}_{n}
$$

and this completes the proof.

\section{Polyhedral products and toric varieties}

In this section, we recall several known results from toric topology.

Definition 3.1 ([3]; Example 6.39). Let $K$ be a simplicial complex on the vertex set $[r]$.

(i) Then we denote by $\mathcal{Z}_{K}$ and $D J(K)$ the moment-angle complex of $K$ and the Davis-Januszkiewicz space of $K$ defined by

$$
\mathcal{Z}_{K}=\mathcal{Z}_{K}\left(D^{2}, S^{1}\right), \quad D J(K)=\mathcal{Z}_{K}\left(\mathbb{C P}^{\infty}, *\right) .
$$

(ii) Let $\iota_{K}: \mathcal{Z}_{K}=\mathcal{Z}_{K}\left(D^{2}, S^{1}\right) \rightarrow D J(K)=\mathcal{Z}_{K}\left(\mathbb{C P}^{\infty}, *\right)$ denote the natural map induced from the following composite of maps

$$
\left(D^{2}, S^{1}\right) \stackrel{\text { pin }}{\longrightarrow}\left(S^{2}, *\right)=\left(\mathbb{C P}^{1}, *\right) \stackrel{\subset}{\longrightarrow}\left(\mathbb{C P}^{\infty}, *\right),
$$

where pin $:\left(D^{2}, S^{1}\right) \rightarrow\left(S^{2}, *\right)$ denotes the natural pinching map. 
Lemma 3.2 ([3], 20]). Let $K$ be a simplicial complex on the vertex set $[r]$.

(i) The space $\mathcal{Z}_{K}$ is 2-connected and there is an $\mathbb{T}^{r}$-equivariant deformation retraction

$$
r_{\mathbb{C}}: U(K)=\mathcal{Z}_{K}\left(\mathbb{C}, \mathbb{C}^{*}\right) \stackrel{\simeq}{\longrightarrow} \mathcal{Z}_{K}, \text { where } \mathbb{T}^{r}=\left(S^{1}\right)^{r} .
$$

(ii) There is a fibration sequence (up to homotopy)

$$
\mathcal{Z}_{K} \stackrel{\iota_{K}}{\longrightarrow} D J(K) \stackrel{\subset}{\longrightarrow}\left(\mathbb{C P}^{\infty}\right)^{r}
$$

(iii) If $X_{\Sigma}$ is a simply connected non-singular toric variety satisfying the condition 2.14.1), there is a fibration sequence (up to homotopy)

$$
\mathbb{T}_{\mathbb{C}}^{n}=\left(\mathbb{C}^{*}\right)^{n} \longrightarrow X_{\Sigma} \stackrel{p_{\Sigma}}{\longrightarrow} D J\left(\mathcal{K}_{\Sigma}\right) .
$$

Proof. The assertions follow from [3, Theorem 6.33, Theorem 8.9], [3, Theorem 6.29, Corollary 6.30] and [20, Proposition 4.4].

Lemma 3.3 ([25]; (6.2) and Proposition 6.7). Let $X_{\Sigma}$ be a non-singular toric variety such that the condition (2.14.2) holds. Then there is an isomorphism

$$
G_{\Sigma} \cong \mathbb{T}_{\mathbb{C}}^{r-n}=\left(\mathbb{C}^{*}\right)^{r-n},
$$

and the group $G_{\Sigma}$ acts on the space $\mathcal{Z}_{\mathcal{K}_{\Sigma}}\left(\mathbb{C}, \mathbb{C}^{*}\right)$ freely. So there is a principal $G_{\Sigma^{-}}$bundle

$$
q_{\Sigma}: U\left(\mathcal{K}_{\Sigma}\right)=\mathcal{Z}_{\mathcal{K}_{\Sigma}}\left(\mathbb{C}, \mathbb{C}^{*}\right) \rightarrow X_{\Sigma}
$$

Lemma 3.4. (i) The space $X_{\Sigma}$ is simply connected if and only if the condition (2.14.2) is satisfied.

(ii) If $X_{\Sigma}$ is a simply connected non-singular toric variety, $\pi_{2}\left(X_{\Sigma}\right)=\mathbb{Z}^{r-n}$ and the map

$$
\Omega q_{\Sigma}: \Omega \mathcal{Z}_{\mathcal{K}_{\Sigma}} \rightarrow \Omega X_{\Sigma}
$$

is a universal covering projection with fiber $\mathbb{Z}^{r-n}$.

Proof. The assertion (i) easily follows from [7, Theorem 12.1.10], and it suffices to show (ii). By Lemma 3.2 and (3.3), $\mathcal{Z}_{\mathcal{K}_{\Sigma}}\left(\mathbb{C}, \mathbb{C}^{*}\right) \simeq \mathcal{Z}_{\mathcal{K}_{\Sigma}}$ is 2-connected. Then by using the homotopy exact sequence of the principal $G_{\Sigma}$-bundle (3.7) and the isomorphism (3.6), we easily see that $\pi_{2}\left(X_{\Sigma}\right)=\mathbb{Z}^{r-n}$ and that $\Omega q_{\Sigma}$ is a universal covering (up to homotopy).

Remark 3.5. If $X_{\Sigma}$ is a simply connected non-singular toric variety satisfying the condition (2.14.1), one can show that there is a homotopy equivalence

$$
\Omega X_{\Sigma} \simeq \Omega \mathcal{Z}_{\mathcal{K}_{\Sigma}} \times \mathbb{T}^{r-n}
$$

Although this can be proved by using (3.3), (3.6) and (3.7), we do not need this fact and omit the detail. 


\section{Simplicial resolutions}

In this section, we summarize the definitions of the non-degenerate simplicial resolution and the associated truncated simplicial resolutions ([22], 27]).

Definition 4.1. (i) For a finite set $\boldsymbol{v}=\left\{v_{1}, \cdots, v_{l}\right\} \subset \mathbb{R}^{N}$, let $\sigma(\boldsymbol{v})$ denote the convex hull spanned by $\boldsymbol{v}$. Let $h: X \rightarrow Y$ be a surjective map such that $h^{-1}(y)$ is a finite set for any $y \in Y$, and let $i: X \rightarrow \mathbb{R}^{N}$ be an embedding. Let $\mathcal{X}^{\Delta}$ and $h^{\Delta}: \mathcal{X}^{\Delta} \rightarrow Y$ denote the space and the map defined by

$$
\mathcal{X}^{\Delta}=\left\{(y, u) \in Y \times \mathbb{R}^{N}: u \in \sigma\left(i\left(h^{-1}(y)\right)\right)\right\} \subset Y \times \mathbb{R}^{N}, h^{\Delta}(y, u)=y .
$$

The pair $\left(\mathcal{X}^{\Delta}, h^{\Delta}\right)$ is called the simplicial resolution of $(h, i)$. In particular, it is called a non-degenerate simplicial resolution if for each $y \in Y$ any $k$ points of $i\left(h^{-1}(y)\right)$ span $(k-1)$-dimensional simplex of $\mathbb{R}^{N}$.

(ii) For each $k \geq 0$, let $\mathcal{X}_{k}^{\Delta} \subset \mathcal{X}^{\Delta}$ be the subspace of the union of the $(k-1)$-skeletons of the simplices over all the points $y$ in $Y$ given by

$$
\mathcal{X}_{k}^{\Delta}=\left\{(y, u) \in \mathcal{X}^{\Delta}: u \in \sigma(\boldsymbol{v}), \boldsymbol{v}=\left\{v_{1}, \cdots, v_{l}\right\} \subset i\left(h^{-1}(y)\right), l \leq k\right\} .
$$

We make the identification $X=\mathcal{X}_{1}^{\Delta}$ by identifying $x \in X$ with the pair $(h(x), i(x)) \in \mathcal{X}_{1}^{\Delta}$, and we note that there is an increasing filtration

$$
\emptyset=\mathcal{X}_{0}^{\Delta} \subset X=\mathcal{X}_{1}^{\Delta} \subset \mathcal{X}_{2}^{\Delta} \subset \cdots \subset \mathcal{X}_{k}^{\Delta} \subset \cdots \subset \bigcup_{k=0}^{\infty} \mathcal{X}_{k}^{\Delta}=\mathcal{X}^{\Delta}
$$

Since the map $h^{\Delta}: \mathcal{X}^{\Delta} \rightarrow Y$ is a proper map, it extends to the map $h_{+}^{\Delta}$ : $\mathcal{X}_{+}^{\Delta} \rightarrow Y_{+}$between the one-point compactifications, where $X_{+}$denotes the one-point compactification of a locally compact space $X$.

(iii) A space $X \subset \mathbb{R}^{n}$ is called semi-algebraic if it is a subspace of the form $X=\bigcup_{i=1}^{s} \bigcap_{j=1}^{r_{i}}\left\{\left(x_{1}, \cdots, x_{n}\right) \in \mathbb{R}^{n}: f_{i j} *_{i j} 0\right\}$, where $f_{i j} \in \mathbb{R}\left[X_{1}, \cdots, x_{n}\right]$ and $*_{i j}$ is either $<$ or $=$, for $i=1, \cdots s$ and $j=1, \cdots, r_{i}$. Similarly, when $X \subset \mathbb{R}^{n}$ and $Y \subset \mathbb{R}^{m}$ are semi-algebraic spaces, a map $f: X \rightarrow Y$ is $a$ semi-algebraic map if the graph of $f$ is semi-algebraic.

Lemma 4.2 ([27], [28] (cf. Lemma 3.3 in [18])). Let $h: X \rightarrow Y$ be a surjective map such that $h^{-1}(y)$ is a finite set for any $y \in Y$, and let $i: X \rightarrow$ $\mathbb{R}^{N}$ be an embedding.

(i) If $X$ and $Y$ are semi-algebraic spaces and the two maps $h$, $i$ are semialgebraic maps, then the map $h_{+}^{\Delta}: \mathcal{X}_{+}^{\Delta} \stackrel{\simeq}{\rightarrow} Y_{+}$is a homotopy equivalence.

(ii) There is an embedding $j: X \rightarrow \mathbb{R}^{M}$ such that the associated simplicial resolution $\left(\tilde{\mathcal{X}}^{\Delta}, \tilde{h}^{\Delta}\right)$ of $(h, j)$ is non-degenerate. 
(iii) If there is an embedding $j: X \rightarrow \mathbb{R}^{M}$ such that the associated simplicial resolution $\left(\tilde{\mathcal{X}}^{\Delta}, \tilde{h}^{\Delta}\right)$ of $(h, j)$ is non-degenerate, the space $\tilde{\mathcal{X}}^{\Delta}$ is uniquely determined up to homeomorphism. Moreover, there is a filtration preserving homotopy equivalence $q^{\Delta}: \tilde{\mathcal{X}}^{\Delta} \stackrel{\simeq}{\rightarrow} \mathcal{X}^{\Delta}$ such that $q^{\Delta} \mid X=i d_{X}$.

Remark 4.3. In this paper we only need the weaker assertion that the map $h_{+}^{\Delta}$ is a homology equivalence. One can easily prove this result by the same argument as used in the second revised edition of Vassiliev's book [27, Proof of Lemma 1 (page 90)].

Remark 4.4 ([27], [28]). Even for a surjective map $h: X \rightarrow Y$ which is not finite to one, it is still possible to construct an associated non-degenerate simplicial resolution. Recall that it is known that there exists a sequence of embeddings $\left\{\tilde{i}_{k}: X \rightarrow \mathbb{R}^{N_{k}}\right\}_{k \geq 1}$ satisfying the following two conditions for each $k \geq 1$ ([27], [28]).

(i) For any $y \in Y$, any $t$ points of the set $\tilde{i}_{k}\left(h^{-1}(y)\right)$ span $(t-1)$-dimensional affine subspace of $\mathbb{R}^{N_{k}}$ if $t \leq 2 k$.

(ii) $N_{k} \leq N_{k+1}$ and if we identify $\mathbb{R}^{N_{k}}$ with a subspace of $\mathbb{R}^{N_{k+1}}$, then $\tilde{i}_{k+1}=\hat{i} \circ \tilde{i}_{k}$, where $\hat{i}: \mathbb{R}^{N_{k}} \subseteq \mathbb{R}^{N_{k+1}}$ denotes the inclusion.

In this situation, in fact, a non-degenerate simplicial resolution may be constructed by choosing a sequence of embeddings $\left\{\tilde{i}_{k}: X \rightarrow \mathbb{R}^{N_{k}}\right\}_{k \geq 1}$ satisfying the above two conditions for each $k \geq 1$.

Let $\mathcal{X}_{k}^{\Delta}=\left\{(y, u) \in Y \times \mathbb{R}^{N_{k}}: u \in \sigma(\boldsymbol{v}), \boldsymbol{v}=\left\{v_{1}, \cdots, v_{l}\right\} \subset \tilde{i}_{k}\left(h^{-1}(y)\right), l \leq\right.$ $k\}$. Then by identifying naturally $\mathcal{X}_{k}^{\Delta}$ with a subspace of $\mathcal{X}_{k+1}^{\Delta}$, define the non-degenerate simplicial resolution $\mathcal{X}^{\Delta}$ of $h$ as $\mathcal{X}^{\Delta}=\bigcup_{k \geq 1} \mathcal{X}_{k}^{\Delta}$.

Definition 4.5. Let $h: X \rightarrow Y$ be a surjective semi-algebraic map between semi-algebraic spaces, $j: X \rightarrow \mathbb{R}^{N}$ be a semi-algebraic embedding, and let $\left(\mathcal{X}^{\Delta}, h^{\Delta}: \mathcal{X}^{\Delta} \rightarrow Y\right)$ denote the associated non-degenerate simplicial resolution of $(h, j)$.

Let $k$ be a fixed positive integer and let $h_{k}: \mathcal{X}_{k}^{\Delta} \rightarrow Y$ be the map defined by the restriction $h_{k}:=h^{\Delta} \mid \mathcal{X}_{k}^{\Delta}$. The fibers of the map $h_{k}$ are $(k-1)$ skeleton of the fibers of $h^{\Delta}$ and, in general, always fail to be simplices over the subspace $Y_{k}=\left\{y \in Y: \operatorname{card}\left(h^{-1}(y)\right)>k\right\}$. Let $Y(k)$ denote the closure of the subspace $Y_{k}$. We modify the subspace $\mathcal{X}_{k}^{\Delta}$ so as to make all the fibers of $h_{k}$ contractible by adding to each fibre of $Y(k)$ a cone whose base is this fibre. We denote by $X^{\Delta}(k)$ this resulting space and by $h_{k}^{\Delta}: X^{\Delta}(k) \rightarrow Y$ the natural extension of $h_{k}$. 
Following [23], we call the map $h_{k}^{\Delta}: X^{\Delta}(k) \rightarrow Y$ the truncated (after the $k$-th term) simplicial resolution of $Y$. Note that that there is a natural filtration

$X_{0}^{\Delta} \subset X_{1}^{\Delta} \subset \cdots \subset X_{l}^{\Delta} \subset X_{l+1}^{\Delta} \subset \cdots \subset X_{k}^{\Delta} \subset X_{k+1}^{\Delta}=X_{k+2}^{\Delta}=\cdots=X^{\Delta}(k)$,

where $X_{0}^{\Delta}=\emptyset, X_{l}^{\Delta}=\mathcal{X}_{l}^{\Delta}$ if $l \leq k$ and $X_{l}^{\Delta}=X^{\Delta}(k)$ if $l>k$.

Lemma 4.6 ([23], cf. Remark 2.4 and Lemma 2.5 in [16]). Under the same assumptions and with the same notation as in Definition 4.5, the map $h_{k}^{\Delta}$ : $X^{\Delta}(k) \stackrel{\simeq}{\longrightarrow} Y$ is a homotopy equivalence.

\section{The Vassiliev spectral sequence}

In this section, we always assume that $D=\left(d_{1}, \cdots, d_{r}\right) \in \mathbb{N}^{r}$ is an $r$-tuple of positive integers and let us write $d_{\min }=\min \left\{d_{1}, \cdots, d_{r}\right\}$. First, we construct the Vassiliev spectral sequence.

Definition 5.1. (i) For a space $X$ and a positive integer $k \geq 1$, let $F(X, k)$ denote the ordered configuration space of $k$-distinct points in $X$ given by

$$
F(X, k)=\left\{\left(x_{1}, \cdots, x_{k}\right) \in X^{k}: x_{i} \neq x_{j} \text { if } i \neq j\right\} .
$$

The symmetric group $S_{k}$ of $k$ letters acts on $F(X, k)$ freely by the usual coordinate permutation and we denote by $C_{k}(X)$ the unordered configuration space of $k$-distinct points in $X$ given by the orbit space

$$
C_{k}(X)=F(X, k) / S_{k}
$$

(ii) Let $\Sigma_{D}$ denote the discriminant of $\operatorname{Pol}_{D}^{*}\left(S^{1}, X_{\Sigma}\right)$ in $\mathrm{P}^{D}$ given by the complement

$$
\begin{aligned}
\Sigma_{D} & =\mathrm{P}^{D} \backslash \operatorname{Pol}_{D}^{*}\left(S^{1}, X_{\Sigma}\right) \\
& =\left\{\left(f_{1}(z), \cdots, f_{r}(z)\right) \in \mathrm{P}^{D}:\left(f_{1}(x), \cdots, f_{r}(x)\right) \in L(\Sigma) \text { for some } x \in \mathbb{R}\right\},
\end{aligned}
$$

where

$$
L(\Sigma)=\bigcup_{\sigma \in I\left(\mathcal{K}_{\Sigma}\right)} L_{\sigma}=\bigcup_{\sigma \subset[r], \sigma \notin K_{\Sigma}} L_{\sigma}
$$

(iii) Let $Z_{D} \subset \Sigma_{D} \times \mathbb{R}$ denote the tautological normalization of $\Sigma_{D}$ consisting of all pairs $\left(\left(f_{1}(z), \ldots, f_{r}(z)\right), x\right) \in \Sigma_{D} \times \mathbb{R}$ satisfying the condition $\left(f_{1}(x), \cdots, f_{r}(x)\right) \in L(\Sigma)$. Projection on the first factor gives a surjective $\operatorname{map} \pi_{D}: Z_{D} \rightarrow \Sigma_{D}$. 
Remark 5.2. Let $\sigma_{k} \in[r]$ for $k=1$,2. It is easy to see that $L_{\sigma_{1}} \subset L_{\sigma_{2}}$ if $\sigma_{1} \supset \sigma_{2}$. Letting

$$
\operatorname{Pr}(\Sigma)=\left\{\sigma=\left\{i_{1}, \cdots, i_{s}\right\} \subset[r]:\left\{\boldsymbol{n}_{i_{1}}, \cdots, \boldsymbol{n}_{i_{s}}\right\} \text { is a primitive in } \Sigma\right\},
$$

we see that

$$
L(\Sigma)=\bigcup_{\sigma \in \operatorname{Pr}(\Sigma)} L_{\sigma}
$$

and by using (2.25) we obtain the equality

$$
\operatorname{dim} L(\Sigma)=2\left(r-r_{\min }(\Sigma)\right)
$$

Our goal in this section is to construct, by means of the non-degenerate simplicial resolution of the discriminant, a spectral sequence converging to the homology of $\operatorname{Pol}_{D}^{*}\left(S^{1}, X_{\Sigma}\right)$.

Definition 5.3. Let $\left(\mathcal{X}^{D}, \pi_{D}^{\Delta}: \mathcal{X}^{D} \rightarrow \Sigma_{D}\right)$ be the non-degenerate simplicial resolution associated to the surjective map $\pi_{D}: Z_{D} \rightarrow \Sigma_{D}$ with the natural increasing filtration as in Definition 4.1,

$$
\emptyset=\mathcal{X}_{0}^{D} \subset \mathcal{X}_{1}^{D} \subset \mathcal{X}_{2}^{D} \subset \cdots \subset \mathcal{X}^{D}=\bigcup_{k=0}^{\infty} \mathcal{X}_{k}^{D}
$$

By Lemma 4.2, the map $\pi_{D}^{\Delta}: \mathcal{X}^{D} \stackrel{\widetilde{\sim}}{\rightarrow} \Sigma_{D}$ is a homotopy equivalence which extends to a homotopy equivalence $\pi_{D+}^{\Delta}: \mathcal{X}_{+}^{D} \stackrel{\simeq}{\rightarrow} \Sigma_{D+}$, where $X_{+}$ denotes the one-point compactification of a locally compact space $X$. Since $\mathcal{X}_{k+}^{D} / \mathcal{X}_{k-1_{+}}^{D} \cong\left(\mathcal{X}_{k}^{D} \backslash \mathcal{X}_{k-1}^{D}\right)_{+}$, we have a spectral sequence

$$
\left\{E_{t ; D}^{k, s}, d_{t}: E_{t ; D}^{k, s} \rightarrow E_{t ; D}^{k+t, s+1-t}\right\} \Rightarrow H_{c}^{k+s}\left(\Sigma_{D} ; \mathbb{Z}\right),
$$

where $E_{1 ; D}^{k, s}=H_{c}^{k+s}\left(\mathcal{X}_{k}^{D} \backslash \mathcal{X}_{k-1}^{D} ; \mathbb{Z}\right)$ and $H_{c}^{k}(X ; \mathbb{Z})$ denotes the cohomology group with compact supports given by $H_{c}^{k}(X, \mathbb{Z})=\tilde{H}^{k}\left(X_{+} ; \mathbb{Z}\right)$.

Let $N(D)$ denote the positive integer given by

$$
N(D)=\sum_{k=1}^{r} d_{k}
$$

Since there is a homeomorphism $\mathrm{P}^{D} \cong \mathbb{C}^{N(D)}$, by Alexander duality there is a natural isomorphism 


$$
\tilde{H}_{k}\left(\operatorname{Pol}_{D}^{*}\left(S^{1}, X_{\Sigma}\right) ; \mathbb{Z}\right) \cong H_{c}^{2 N(D)-k-1}\left(\Sigma_{D} ; \mathbb{Z}\right) \text { for any } k .
$$

By reindexing we obtain a spectral sequence

$$
\left\{E_{k, s}^{t ; D}, \tilde{d}^{t}: E_{k, s}^{t ; D} \rightarrow E_{k+t, s+t-1}^{t ; D}\right\} \Rightarrow H_{s-k}\left(\mathrm{Pol}_{D}^{*}\left(S^{1}, X_{\Sigma}\right) ; \mathbb{Z}\right),
$$

where $E_{k, s}^{1 ; D}=H_{c}^{2 N(D)+k-s-1}\left(\mathcal{X}_{k}^{D} \backslash \mathcal{X}_{k-1}^{D} ; \mathbb{Z}\right)$.

Let $L_{k ; \Sigma} \subset(\mathbb{R} \times L(\Sigma))^{k}$ denote the subspace defined by

$$
L_{k ; \Sigma}=\left\{\left(\left(x_{1}, s_{1}\right), \cdots,\left(x_{k}, s_{k}\right)\right): x_{j} \in \mathbb{R}, s_{j} \in L(\Sigma), x_{i} \neq x_{j} \text { if } i \neq j\right\} .
$$

The symmetric group $S_{k}$ on $k$ letters acts on $L_{k ; \Sigma}$ by permuting coordinates. Let $C_{k ; \Sigma}$ denote the orbit space

$$
C_{k ; \Sigma}=L_{k ; \Sigma} / S_{k}
$$

By (5.5) we know that $C_{k ; \Sigma}$ is a cell-complex of dimension

$$
\operatorname{dim} C_{k ; \Sigma}=\left(1+2 r-2 r_{\min }(\Sigma)\right) k .
$$

Lemma 5.4. If $1 \leq k \leq d_{\min }=\min \left\{d_{1}, \cdots, d_{r}\right\}, \mathcal{X}_{k}^{D} \backslash \mathcal{X}_{k-1}^{D}$ is homeomorphic to the total space of a real affine bundle $\xi_{D, k}$ over $C_{k ; \Sigma}$ with rank $l_{D, k}=$ $2 N(D)-2 r k+k-1$.

Proof. The argument is exactly analogous to the one in the proof of [1, Lemma 4.4]. Namely, an element of $\mathcal{X}_{k}^{D} \backslash \mathcal{X}_{k-1}^{D}$ is represented by $(F, u)=$ $\left(\left(f_{1}, \cdots, f_{r}\right), u\right)$, where $F=\left(f_{1}, \cdots, f_{r}\right)$ is an $r$-tuple of monic polynomials in $\Sigma_{D}$ and $u$ is an element of the interior of the span of the images of $k$ distinct points $\left\{x_{1}, \cdots, x_{k}\right\} \in C_{k}(\mathbb{R})$ such that $F\left(x_{j}\right)=\left(f_{1}\left(x_{j}\right), \cdots, f_{r}\left(x_{j}\right)\right) \in L(\Sigma)$ for each $1 \leq j \leq k$, under a suitable embedding. Since the $k$ distinct points $\left\{x_{j}\right\}_{j=1}^{k}$ are uniquely determined by $u$, by the definition of the non-degenerate simplicial resolution, there are projection maps $\pi_{k, D}: \mathcal{X}_{k}^{D} \backslash \mathcal{X}_{k-1}^{D} \rightarrow C_{k ; \Sigma}$ given by $\left(\left(f_{1}, \cdots, f_{r}\right), u\right) \mapsto\left\{\left(x_{1}, F\left(x_{1}\right)\right), \ldots,\left(x_{k}, F\left(x_{k}\right)\right)\right\}$.

Now suppose that $1 \leq k \leq d_{\text {min }}$ and let $c=\left\{\left(x_{j}, s_{j}\right)\right\}_{j=1}^{k} \in C_{k ; \Sigma}\left(x_{j} \in \mathbb{R}\right.$, $s_{j} \in L(\Sigma)$ ) be any fixed element. Consider the fibre $\pi_{k, D}^{-1}(c)$. For each $1 \leq j \leq k$, we set $s_{j}=\left(s_{1, j}, \cdots, s_{r, j}\right)$ and consider the condition

$$
F\left(x_{j}\right)=\left(f_{1}\left(x_{j}\right), \cdots, f_{r}\left(x_{j}\right)\right)=s_{j} \Leftrightarrow f_{t}\left(x_{j}\right)=s_{t, j} \quad \text { for } 1 \leq t \leq r .
$$

In general, the condition $f_{t}\left(x_{j}\right)=s_{t, j}$ gives one linear condition on the coefficients of $f_{t}$, and determines an affine hyperplane in $\mathrm{P}^{d_{t}}(\mathbb{C})$. For example, if 
we set $f_{t}(z)=z^{d_{t}}+\sum_{i=0}^{d_{t}-1} a_{i, t} z^{i}$, then $f_{t}\left(x_{j}\right)=s_{t, j}$ for any $1 \leq j \leq k$ if and only if

$$
\left[\begin{array}{ccccc}
1 & x_{1} & x_{1}^{2} & \cdots & x_{1}^{d_{t}-1} \\
1 & x_{2} & x_{2}^{2} & \cdots & x_{2}^{d_{t}-1} \\
\vdots & \ddots & \ddots & \ddots & \vdots \\
1 & x_{k} & x_{k}^{2} & \cdots & x_{k}^{d_{t}-1}
\end{array}\right] \cdot\left[\begin{array}{c}
a_{0, t} \\
a_{1, t} \\
\vdots \\
a_{d_{t}-1, t}
\end{array}\right]=\left[\begin{array}{c}
s_{t, 1}-x_{1}^{d_{t}} \\
s_{t, 2}-x_{2}^{d_{t}} \\
\vdots \\
s_{t, k}-x_{k}^{d_{t}}
\end{array}\right]
$$

Since $1 \leq k \leq d_{\min }(D)$ and $\left\{x_{j}\right\}_{j=1}^{k} \in C_{k}(\mathbb{R})$, it follows from the properties of Vandermonde matrices that the condition (5.13) gives exactly $k$ independent conditions on the coefficients of $f_{t}(z)$. Thus the space of polynomials $f_{t}(z)$ in $\mathrm{P}^{d_{t}}(\mathbb{C})$ which satisfies (5.13) is the intersection of $k$ affine hyperplanes in general position and has codimension $k$ in $\mathrm{P}^{d_{t}}(\mathbb{C})$. Hence, the fibre $\pi_{k, D}^{-1}(c)$ is homeomorphic to the product of an open $(k-1)$-simplex with the real affine space of dimension $2 \sum_{i=1}^{r}\left(d_{i}-k\right)=2 N(D)-2 r k$. It is now easy to show that $\pi_{k, D}$ is a (locally trivial) real affine bundle over $C_{k ; \Sigma}$ of rank $l_{D, k}=2 N(D)-2 r k+k-1$.

Lemma 5.5. If $1 \leq k \leq d_{\min }$, there is a natural isomorphism

$$
E_{k, s}^{1 ; D} \cong H_{c}^{2 r k-s}\left(C_{k ; \Sigma} ; \pm \mathbb{Z}\right),
$$

where the twisted coefficients system $\pm \mathbb{Z}$ comes from the Thom isomorphism.

Proof. Suppose that $1 \leq k \leq d_{\min }$. By Lemma 5.4, there is a homeomorphism $\left(\mathcal{X}_{k}^{D} \backslash \mathcal{X}_{k-1}^{D}\right)_{+} \cong T\left(\xi_{D, k}\right)$, where $T\left(\xi_{D, k}\right)$ denotes the Thom space of $\xi_{D, k}$. Since $(2 N(D)+k-s-1)-l_{D, k}=2 r k-s$, by using the Thom isomorphism there is a natural isomorphism $E_{k, s}^{1 ; D} \cong H_{c}^{2 r k-s}\left(C_{k ; \Sigma} ; \pm \mathbb{Z}\right)$.

Definition 5.6. (i) Let $X^{\Delta}$ denote the truncated (after the $d_{\min }$-th term) simplicial resolution of $\Sigma_{D}$ with the natural filtration

$$
\emptyset=X_{0}^{\Delta} \subset X_{1}^{\Delta} \subset \cdots \subset X_{d_{\min }}^{\Delta} \subset X_{d_{\min }+1}^{\Delta}=X_{d_{\min }+2}^{\Delta}=\cdots=X^{\Delta},
$$

where $X_{k}^{\Delta}=\mathcal{X}_{k}^{D}$ if $k \leq d_{\min }$ and $X_{k}^{\Delta}=X_{d_{\min }+1}^{\Delta}$ if $k \geq d_{\min }+1$.

(ii) Let $\left\{\boldsymbol{e}_{i}: 1 \leq i \leq r\right\}$ denote the standard $\mathbb{R}$-basis of $\mathbb{R}^{r}$ given by

$$
\boldsymbol{e}_{1}=(1,0,0, \cdots 0), \boldsymbol{e}_{2}=(0,1,0, \cdots 0), \cdots, \boldsymbol{e}_{r}=(0, \cdots, 0,0,1) \in \mathbb{R}^{r}
$$

For each $1 \leq i \leq r$, let us consider

$$
D+\boldsymbol{e}_{i}=\left(d_{1}, \cdots, d_{i-1}, d_{i}+1, d_{i+1}, \cdots, d_{r}\right)
$$


and let $Y^{\Delta}$ denote the truncated (after the $d_{\text {min }}$-th term) simplicial resolution of $\Sigma_{D+e_{i}}$ with the natural filtration

$$
\emptyset=Y_{0}^{\Delta} \subset Y_{1}^{\Delta} \subset \cdots \subset Y_{d_{\min }}^{\Delta} \subset Y_{d_{\min }+1}^{\Delta}=Y_{d_{\min }+2}^{\Delta}=\cdots=Y^{\Delta},
$$

where $Y_{k}^{\Delta}=\mathcal{X}_{k}^{D}$ if $k \leq d_{\min }$ and $Y_{k}^{\Delta}=Y^{\Delta}$ if $k \geq d_{\min }+1$.

Then by using the same argument as in (5.8) and Lemma 4.6, we have the following two truncated spectral sequences

$$
\begin{aligned}
& \left\{E_{k, s}^{t}, d^{t}: E_{k, s}^{t} \rightarrow E_{k+t, s+t-1}^{t}\right\} \quad \Rightarrow H_{s-k}\left(\mathrm{Pol}_{D}^{*}\left(S^{1} . X_{\Sigma}\right) ; \mathbb{Z}\right) \\
& \left\{{ }^{\prime} E_{k, s}^{t},{ }^{\prime} d^{t}:{ }^{\prime} E_{k, s}^{t} \rightarrow{ }^{\prime} E_{k+t, s+t-1}^{t}\right\} \Rightarrow H_{s-k}\left(\mathrm{Pol}_{D+e_{i}}^{*}\left(S^{1}, X_{\Sigma}\right) ; \mathbb{Z}\right),
\end{aligned}
$$

where

$$
E_{k, s}^{1}=H_{c}^{2 N(D)+k-s-1}\left(X_{k}^{\Delta} \backslash X_{k-1}^{\Delta} ; \mathbb{Z}\right), E_{k, s}^{1}=H_{c}^{2 N(D)+k-s+1}\left(Y_{k}^{\Delta} \backslash Y_{k-1}^{\Delta} ; \mathbb{Z}\right) .
$$

Lemma 5.7. (i) If $k<0$ or $k \geq d_{\min }+2, E_{k, s}^{1}={ }^{\prime} E_{k, s}^{1}=0$ for any $s$.

(ii) $E_{0,0}^{1}={ }^{\prime} E_{0,0}^{1}=\mathbb{Z}$ and $E_{0, s}^{1}={ }^{\prime} E_{0, s}^{1}=0$ if $s \neq 0$.

(iii) If $1 \leq k \leq d_{\min }$, there are isomorphisms

$$
E_{k, s}^{1} \cong{ }^{\prime} E_{k, s}^{1} \cong H_{c}^{2 r k-s}\left(C_{k ; \Sigma} ; \pm \mathbb{Z}\right) .
$$

(iv) If $1 \leq k \leq d_{\text {min }}, E_{k, s}^{1}={ }^{\prime} E_{k, s}^{1}=0$ for any $s \leq\left(2 r_{\min }(\Sigma)-1\right) k-1$.

(v) $E_{d_{\min }+1, s}^{1}={ }^{\prime} E_{d_{\min }+1, s}^{1}=0$ for any $s \leq\left(2 r_{\min }(\Sigma)-1\right) d_{\min }-1$.

Proof. Let us write $r_{\min }=r_{\min }(\Sigma)$. Since the proofs of both cases are almost identical, it suffices to prove the assertions for the case $E_{k, s}^{1}$. Since $X_{k}^{\Delta}=\mathcal{X}_{k}^{D}$ for any $k \geq d_{\min }+2$, the assertions (i) and (ii) are clearly true. Since $X_{k}^{\Delta}=\mathcal{X}_{k}^{D}$ for any $k \leq d_{\text {min }}$, (iii) easily follows from Lemma 5.5. Thus it remains to prove the assertions (iv) and (v).

By using (5.11), we see that $2 r k-s>\operatorname{dim} C_{k ; \Sigma}$ if and only if $s \leq$ $\left(2 r_{\min }(\Sigma)-1\right) k-1$. Thus $H_{c}^{2 r k-s}\left(C_{k ; \Sigma} ; \pm \mathbb{Z}\right)=0$ if $s \leq\left(2 r_{\min }(\Sigma)-1\right) k-1$ and the assertion (iv) follows from (iii).

Finally consider the group $E_{d_{\min }+1, s}^{1}$. Then by Lemma [23, Lemma 2.1],

$$
\begin{aligned}
\operatorname{dim}\left(X_{d_{\min }+1}^{\Delta} \backslash X_{d_{\min }}^{\Delta}\right) & =\operatorname{dim}\left(\mathcal{X}_{d_{\min }}^{D} \backslash \mathcal{X}_{d_{\min }-1}^{D}\right)+1=\left(l_{D, d_{\min }}+\operatorname{dim} C_{d_{\min } ; \Sigma}\right)+1 \\
& =2 N(D)+2 d_{\min }-2 r_{\min } d_{\min } .
\end{aligned}
$$

Since $E_{d_{\min }+1, s}^{1}=H_{c}^{2 N(D)+d_{\min }-s}\left(X_{d_{\min }+1}^{\Delta} \backslash X_{d_{\min }}^{\Delta} ; \mathbb{Z}\right)$ and $2 N(D)+d_{\min }-s>$ $\operatorname{dim}\left(X_{d+1}^{\Delta} \backslash X_{d}^{\Delta}\right) \Leftrightarrow s \leq\left(2 r_{\min }-1\right) d_{\min }-1$, we see that $E_{d_{\min }+1, s}^{1}=0$ for any $s \leq\left(2 r_{\min }-1\right) d_{\min }-1$. Hence, $(\mathrm{v})$ is proved. 
Definition 5.8. Define the integer $C_{D}(\Sigma)$ by

$$
C_{D}(\Sigma)=2\left(r_{\min }(\Sigma)-1\right) d_{\min }-1 \text {. }
$$

Note that $r_{\min }(\Sigma) \geq 2$ and $d_{\min }(D) \geq 1$ for any $D \in \mathbb{N}^{r}$. Thus, we see that $C_{D}(\Sigma) \geq 1$ for any $D \in \mathbb{N}^{r}$.

Proposition 5.9. The space $\operatorname{Pol}_{D}^{*}\left(S^{1}, X_{\Sigma}\right)$ is $C_{D}(\Sigma)$-connected. Thus, the space $\operatorname{Pol}_{D}^{*}\left(S^{1}, X_{\Sigma}\right)$ is simply connected for any $D \in \mathbb{N}^{r}$.

Proof. it follows from Lemma 5.7 that we can easily check that $E_{k, s}^{1}=0$ if $s-k \leq C_{D}(\Sigma)=2\left(r_{\min }(\Sigma)-1\right) d_{\min }-1$. Hence by using the first spectral sequence given in (5.16), we see that $H_{i}\left(\operatorname{Pol}_{D}^{*}\left(S^{1}, X_{\Sigma}\right) ; \mathbb{Z}\right)=0$ for any $1 \leq$ $i \leq C_{D}(\Sigma)$.

On the other hand, by using the string representation as in [12, Appendix], one can show that $\pi_{1}\left(\operatorname{Pol}_{D}^{*}\left(S^{1}, X_{\Sigma}\right)\right)$ is an abelian group and one know that there is an isomorphism $\pi_{1}\left(\operatorname{Pol}_{D}^{*}\left(S^{1}, X_{\Sigma}\right)\right) \cong H_{1}\left(\operatorname{Pol}_{D}^{*}\left(S^{1}, X_{\Sigma}\right) ; \mathbb{Z}\right)=0$. Thus, the space $\operatorname{Pol}_{D}^{*}\left(S^{1}, X_{\Sigma}\right)$ is simply connected and it follows from the Hurewicz theorem that the space $\operatorname{Pol}_{D}^{*}\left(S^{1}, X_{\Sigma}\right)$ is $C_{D}(\Sigma)$-connected.

\section{$6 \quad$ Stabilization maps}

Definition 6.1. For an $r$-tuple $D=\left(d_{1}, \cdots, d_{r}\right) \in \mathbb{N}^{r}$ of positive integers, let $N(D)$ denote the positive integer given by $N(D)=\sum_{k=1}^{r} d_{k}$ as in (5.6), and let us write $U_{D}=\{w \in \mathbb{C}: \operatorname{Re}(w)<N(D)\}$. For each $D=\left(d_{1}, \cdots, d_{r}\right) \in \mathbb{N}^{r}$ let us choose any any homeomorphism

$$
\varphi_{D}: \mathbb{C} \stackrel{\cong}{\longrightarrow} U_{D}
$$

such that $\varphi_{D}(\mathbb{R})=\{w \in \mathbb{R}: w<N(D)\}=(-\infty, N(D))$ and fix it.

(i) For a monic polynomial $f(z)=\prod_{k=1}^{d}\left(z-\alpha_{k}\right) \in \mathrm{P}^{d}(\mathbb{C})$, let $\varphi_{D}(f(z))$ denote the monic polynomial of the same degree $d$ defined by

$$
\varphi_{D}(f(z))=\prod_{k=1}^{d}\left(z-\varphi\left(\alpha_{k}\right)\right)
$$

(ii) Now we choose a point $x_{D} \in \mathbb{C} \backslash \overline{U_{D}}=\{w \in \mathbb{C}: \operatorname{Re}(w)>N(D)\}$ freely and fix it. Then for each $1 \leq i \leq r$, let us recall

$$
D+\boldsymbol{e}_{i}=\left(d_{1}, \cdots, d_{i-1}, d_{i}+1, d_{i+1}, \cdots, d_{r}\right)
$$


and define the stabilization map

$$
\begin{gathered}
s_{D, i}: \operatorname{Pol}_{D}^{*}\left(S^{1}, X_{\Sigma}\right) \rightarrow \operatorname{Pol}_{D+e_{i}}^{*}\left(S^{1}, X_{\Sigma}\right) \quad \text { by } \\
s_{D, i}(f)=\left(\varphi_{D}\left(f_{1}(z)\right), \cdots, \varphi_{D}\left(f_{i}(z)\right)\left(z-x_{D}\right), \cdots, \varphi_{D}\left(f_{r}(z)\right)\right)
\end{gathered}
$$

for $f=\left(f_{1}(z), \cdots, f_{r}(z)\right) \in \operatorname{Pol}_{D}^{*}\left(S^{1}, X_{\Sigma}\right)$.

(iii) Let $D=\left(d_{1}, \cdots, d_{r}\right) \in \mathbb{N}^{r}$ and $D^{\prime}=\left(d_{1}^{\prime}, \cdots, d_{r}^{\prime}\right) \in \mathbb{N}^{r}$ be $r$-tuples of positive integers such that $D^{\prime}-D=\left(d_{1}^{\prime}-d_{1}, \cdots, d_{r}^{\prime}-d_{r}\right) \in(\mathbb{N} \cup\{0\})^{r} \backslash\left\{\mathbf{0}_{r}\right\}$. Now let us choose any point $\boldsymbol{x}_{D}=\left(x_{D, 1}, \cdots, x_{D, r}\right) \in F\left(\mathbb{C} \backslash \overline{U_{D}}, r\right)$.

Then define the stabilization map

$$
s_{D, D^{\prime}}: \operatorname{Pol}_{D}^{*}\left(S^{1}, X_{\Sigma}\right) \rightarrow \operatorname{Pol}_{D^{\prime}}^{*}\left(S^{1}, X_{\Sigma}\right) \quad \text { by }
$$

$$
s_{D}(f)=\left(\left(\varphi_{D}\left(f_{1}(z)\right)\left(z-x_{D, 1}\right)^{d_{1}^{\prime}-d_{1}}, \cdots, \varphi_{D}\left(f_{r}(z)\right)\left(z-x_{D, r}\right)^{d_{r}^{\prime}-d_{r}}\right)\right.
$$

for $f=\left(f_{1}(z), \cdots, f_{r}(z)\right) \in \operatorname{Pol}_{D}^{*}\left(S^{1}, X_{\Sigma}\right)$. In particular, when $D^{\prime}=D+\boldsymbol{e}=$ $\left(d_{1}+1, d_{2}+1, \cdots, d_{r}+1\right)$, we write

$$
s_{D}=s_{D, D^{\prime}}=s_{D, D+e}: \operatorname{Pol}_{D}^{*}\left(S^{1}, X_{\Sigma}\right) \rightarrow \operatorname{Pol}_{D+e}^{*}\left(S^{1}, X_{\Sigma}\right),
$$

where we set

$$
\boldsymbol{e}=(1,1, \cdots, 1)=\boldsymbol{e}_{1}+\boldsymbol{e}_{2}+\cdots+\boldsymbol{e}_{r}
$$

Remark 6.2. Note that the definition of the map $s_{D, i}$ depends on the choice of the pair $\left(\varphi_{D}, x_{D}\right)$, but the homotopy class of the map $s_{D, i}$ does not depend on its choice. Similarly the definition of the map $s_{D, D^{\prime}}$ also depends on the choice of the pair $\left(\varphi_{d}, \boldsymbol{x}_{D}\right)$, but its homotopy class does not.

Now recall the definition of the stabilization map

$$
s_{D, i}: \operatorname{Pol}_{D}^{*}\left(S^{1}, X_{\Sigma}\right) \rightarrow \operatorname{Pol}_{D+e_{i}}^{*}\left(S^{1}, X_{\Sigma}\right) .
$$

By using the choice of the point $x_{D} \in \mathbb{C} \backslash \overline{U_{D}}$, one can easily see that it extends to an open embedding

$$
\bar{s}_{D, i}: \mathbb{C} \times \operatorname{Pol}_{D}^{*}\left(S^{1}, X_{\Sigma}\right) \rightarrow \operatorname{Pol}_{D+e_{i}}^{*}\left(S^{1}, X_{\Sigma}\right) .
$$

It also naturally extends to an open embedding $\tilde{s}_{D, i}: \mathrm{P}^{D} \rightarrow \mathrm{P}^{D_{i}}$ and by restriction we obtain an open embedding

$$
\tilde{s}_{D, i}: \mathbb{C} \times \Sigma_{D} \rightarrow \Sigma_{D+e_{i}} .
$$


Since one-point compactification is contravariant for open embeddings, this map induces a map $\left(\tilde{s}_{D+e_{i}}\right)_{+}:\left(\Sigma_{D_{i}}\right)_{+} \rightarrow\left(\mathbb{C} \times \Sigma_{D}\right)_{+}=S^{2} \wedge \Sigma_{D+}$.

Note that there is a commutative diagram

$$
\begin{array}{cc}
\tilde{H}_{k}\left(\mathrm{Pol}_{D}^{*}\left(S^{1}, X_{\Sigma}\right) ; \mathbb{Z}\right) \stackrel{s_{D, i_{*}}}{\longrightarrow} \tilde{H}_{k}\left(\mathrm{Pol}_{D+e_{i}}^{*}\left(S^{1}, X_{\Sigma}\right) ; \mathbb{Z}\right) \\
A l \downarrow \cong & A l \cong \\
\downarrow H_{c}^{2 N(D)-k-1}\left(\Sigma_{D} ; \mathbb{Z}\right) & \stackrel{\tilde{s}_{D_{i}+}^{*}}{\longrightarrow} H_{c}^{2 N(D)-k+1}\left(\Sigma_{D+e_{i}} ; \mathbb{Z}\right)
\end{array}
$$

where $A l$ is the Alexander duality isomorphism and $\tilde{s}_{D_{i}+}^{*}$ denotes the composite of the suspension isomorphism with the homomorphism $\left(\tilde{s}_{D+}\right)^{*}$,

$H_{c}^{2 N(D)-k-1}\left(\Sigma_{D} ; \mathbb{Z}\right) \stackrel{\cong}{\rightarrow} H_{c}^{2 N(D)-k+1}\left(\mathbb{C} \times \Sigma_{D} ; \mathbb{Z}\right) \stackrel{\left(\tilde{s}_{D_{i}+}\right)^{*}}{\longrightarrow} H_{c}^{2 N(D)-k+1}\left(\Sigma_{D+e_{i}} ; \mathbb{Z}\right)$.

By the universality of the non-degenerate simplicial resolution ([22, pages 286-287]), the map $\tilde{s}_{D, i}$ also naturally extends to a filtration preserving open embedding

$$
\tilde{s}_{D, i}: \mathbb{C} \times \mathcal{X}^{D} \rightarrow \mathcal{X}^{D+e_{i}}
$$

between non-degenerate simplicial resolutions. This induces a filtration preserving map $\left(\tilde{s}_{D, i}\right)_{+}: \mathcal{X}_{+}^{D+e_{i}} \rightarrow\left(\mathbb{C} \times \mathcal{X}^{D}\right)_{+}=S^{2} \wedge \mathcal{X}_{+}^{D}$, and we obtain the homomorphism of spectral sequences

$$
\left\{\tilde{\theta}_{k, s}^{t}: E_{k, s}^{t ; D} \rightarrow E_{k, s}^{t ; D+e_{i}}\right\}, \quad \text { where }
$$

$$
\begin{aligned}
\left\{E_{k, s}^{t ; D}, \tilde{d}^{t}: E_{k, s}^{t ; D} \rightarrow E_{k+t, s+t-1}^{t ; D}\right\} & \Rightarrow H_{s-k}\left(\operatorname{Pol}_{D}^{*}\left(S^{1}, X_{\Sigma}\right) ; \mathbb{Z}\right), \\
\left\{E_{k, s}^{t ; D+e_{i}}, \tilde{d}^{t}: E_{k, s}^{t ; D+e_{i}} \rightarrow E_{k+t, s+t-1}^{t ; D+e_{i}}\right\} & \Rightarrow H_{s-k}\left(\operatorname{Pol}_{D+e_{i}}^{*}\left(S^{1}, X_{\Sigma}\right) ; \mathbb{Z}\right),
\end{aligned}
$$

and the groups $E_{k, s}^{1 ; D}$ and $E_{k, s}^{1 ; D_{i}}$ are given by

$$
\left\{\begin{array}{l}
E_{k, s}^{1 ; D}=H_{c}^{2 N(D)+k-1-s}\left(\mathcal{X}_{k}^{D} \backslash \mathcal{X}_{k-1}^{D} ; \mathbb{Z}\right), \\
E_{k, s}^{1 ; D+e_{i}}=H_{c}^{2 N(D)+k+1-s}\left(\mathcal{X}_{k}^{D+e_{i}} \backslash \mathcal{X}_{k-1}^{D+e_{i}} ; \mathbb{Z}\right) .
\end{array}\right.
$$

Lemma 6.3. If $1 \leq i \leq r$ and $0 \leq k \leq d_{\min }, \tilde{\theta}_{k, s}^{1}: E_{k, s}^{1 ; D} \rightarrow E_{k, s}^{1 ; D+e_{i}}$ is an isomorphism for any $s$.

Proof. Since the case $k=0$ is clear, suppose that $1 \leq k \leq d_{\text {min }}$. It follows from the proof of Lemma 5.4 that there is a homotopy commutative diagram of affine vector bundles

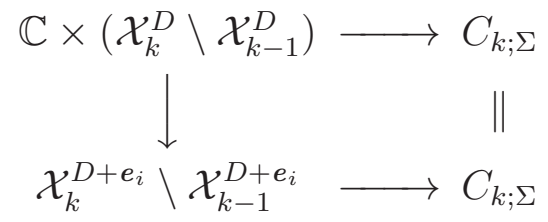


Hence, we have a commutative diagram

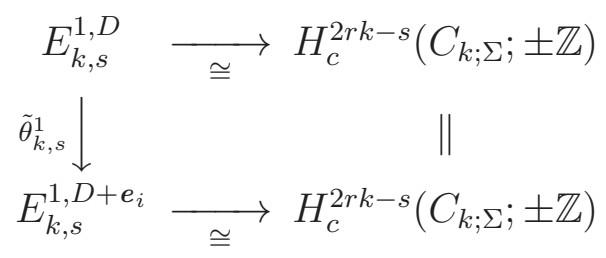

and the assertion follows.

Now we consider the spectral sequences induced by truncated simplicial resolutions.

By using Lemma 4.6] and the same method as in [23, $\S 2$ and $\S 3$ ] (cf. [16. Lemma 2.2]), we obtain the following truncated spectral sequences

$$
\begin{aligned}
\left\{E_{k, s}^{t}, d^{t}: E_{k, s}^{t} \rightarrow E_{k+t, s+t-1}^{t}\right\} & \Rightarrow H_{s-k}\left(\mathrm{Pol}_{D}^{*}\left(S^{1}, X_{\Sigma}\right) ; \mathbb{Z}\right), \\
\left\{{ }^{\prime} E_{k, s}^{t}, d^{t}:{ }^{\prime} E_{k, s}^{t} \rightarrow{ }^{\prime} E_{k+t, s+t-1}^{t}\right\} & \Rightarrow H_{s-k}\left(\mathrm{Pol}_{D+e_{i}}^{*}\left(S^{1}, X_{\Sigma}\right) ; \mathbb{Z}\right),
\end{aligned}
$$

where

$$
E_{k, s}^{1}=H_{c}^{2 N(D)+k-1-s}\left(X_{k}^{\Delta} \backslash X_{k-1}^{\Delta} ; \mathbb{Z}\right),{ }^{\prime} E_{k, s}^{1}=H_{c}^{2(N(D)+k+1-s}\left(Y_{k}^{\Delta} \backslash Y_{k-1}^{\Delta} ; \mathbb{Z}\right) .
$$

By the naturality of truncated simplicial resolutions, the filtration preserving map $\tilde{s}_{D, i}: \mathbb{C} \times \mathcal{X}^{D} \rightarrow \mathcal{X}^{D_{i}}$ gives rise to a natural filtration preserving map $\tilde{s}_{D, i}^{\prime}: \mathbb{C} \times X^{\Delta} \rightarrow Y^{\Delta}$ which, in a way analogous to (6.12), induces a homomorphism of spectral sequences

$$
\left\{\theta_{k, s}^{t}: E_{k, s}^{t} \rightarrow{ }^{\prime} E_{k, s}^{t}\right\} .
$$

Lemma 6.4. If $0 \leq k \leq d_{\min }, \theta_{k, s}^{1}: E_{k, s}^{1} \rightarrow{ }^{\prime} E_{k, s}^{1}$ is an isomorphism for any $s$.

Proof. Since $\left(X_{k}^{\Delta}, Y_{k}^{\Delta}\right)=\left(\mathcal{X}_{k}^{D}, \mathcal{X}_{k}^{D_{i}}\right)$ for $k \leq d_{\text {min }}$, the assertion follows from Lemma 6.3.

Theorem 6.5. For each $1 \leq i \leq r$ and $D=\left(d_{1}, \cdots, d_{r}\right) \in \mathbb{N}^{r}$, the stabilization map

$$
s_{D, i}: \operatorname{Pol}_{D}^{*}\left(S^{1}, X_{\Sigma}\right) \rightarrow \operatorname{Pol}_{D+e_{i}}^{*}\left(S^{1}, X_{\Sigma}\right)
$$

is a homotopy equivalence through dimension $d(D, \Sigma)$, where the number $d(D, \Sigma)$ is given by $d(D, \Sigma)=2\left(r_{\min }(\Sigma)-1\right) d_{\min }-2$ as in (2.25).

Proof. We write $r_{\text {min }}=r_{\text {min }}(\Sigma)$. Since $\operatorname{Pol}_{D}^{*}\left(S^{1}, X_{\Sigma}\right)$ and $\operatorname{Pol}_{D+\boldsymbol{e}_{i}}^{*}\left(S^{1}, X_{\Sigma}\right)$ are simply connected, it suffices to show that the map $s_{D, i}$ is a homology equivalence through dimension $d(D, \Sigma)$. 
For this purpose, consider the homomorphism $\theta_{k, s}^{t}: E_{k, s}^{t} \rightarrow{ }^{\prime} E_{k, s}^{t}$ of truncated spectral sequences given in (6.13). By using the commutative diagram (6.11) and the comparison theorem for spectral sequences, it suffices to prove that the positive integer $d(D, \Sigma)$ has the following property:

(*) $\theta_{k, s}^{\infty}$ is an isomorphism for all $(k, s)$ such that $s-k \leq d(D, \Sigma)$.

By Lemma 5.7, $E_{k, s}^{1}={ }^{\prime} E_{k, s}^{1}=0$ if $k<0$, or if $k \geq d_{\min }+2$, or if $k=d_{\min }+1$ with $s \leq\left(2 r_{\min }-2\right) d_{\min }-1$. Since $\left(2 r_{\min }-1\right) d_{\min }-1-\left(d_{\min }+1\right)=$ $\left(2 r_{\min }-2\right) d_{\min }-2=d(D, \Sigma)$, we see that:

$(*)_{1}$ if $k<0$ or $k \geq d_{\text {min }}+1, \theta_{k, s}^{\infty}$ is an isomorphism for all $(k, s)$ such that $s-k \leq d(D, \Sigma)$.

Next, we assume that $0 \leq k \leq d_{\min }$, and investigate the condition that $\theta_{k, s}^{\infty}$ is an isomorphism. Now recall that $E_{k, s}^{1}={ }^{\prime} E_{k, s}^{1}=0$ if $1 \leq k \leq d_{\text {min }}$ with $s \leq\left(2 r_{\min }-1\right) k-1$ or if $k=0$ with $s \neq 0$. (by Lemma 5.7).

Note that the groups $E_{k_{1}, s_{1}}^{1}$ and ' $E_{k_{1}, s_{1}}^{1}$ are not known for $(u, v) \in \mathcal{S}_{1}=$ $\left\{\left(d_{\min }+1, s\right) \in \mathbb{Z}^{2}: s \geq\left(2 r_{\min }-1\right) d_{\min }\right\}$. By considering the differentials $d^{1}$ 's of $E_{k, s}^{1}$ and ' $E_{k, s}^{1}$, and applying Lemma 6.4, we see that $\theta_{k, s}^{2}$ is an isomorphism if $(k, s) \notin \mathcal{S}_{1} \cup \mathcal{S}_{2}$, where

$$
\mathcal{S}_{2}=\left\{(u, v) \in \mathbb{Z}^{2}:(u+1, v) \in \mathcal{S}_{1}\right\}=\left\{\left(d_{\min }, v\right) \in \mathbb{Z}^{2}: v \geq\left(2 r_{\min }-1\right) d_{\min }\right\} .
$$

A similar argument shows that $\theta_{k, s}^{3}$ is an isomorphism if $(k, s) \notin \bigcup_{t=1}^{3} \mathcal{S}_{t}$, where $\mathcal{S}_{3}=\left\{(u, v) \in \mathbb{Z}^{2}:(u+2, v+1) \in \mathcal{S}_{1} \cup \mathcal{S}_{2}\right\}$. Continuing in the same fashion, considering the differentials $d^{t}$ 's on $E_{k, s}^{t}$ and ' $E_{k, s}^{t}$ and applying the inductive hypothesis, we see that $\theta_{k, s}^{\infty}$ is an isomorphism if $(k, s) \notin \mathcal{S}:=$ $\bigcup_{t \geq 1} \mathcal{S}_{t}=\bigcup_{t \geq 1} A_{t}$, where $A_{t}$ denotes the set

$$
A_{t}=\left\{\begin{array}{l|l}
(u, v) \in \mathbb{Z}^{2} & \begin{array}{l}
\text { There are positive integers } l_{1}, \cdots, l_{t} \text { such that } \\
1 \leq l_{1}<l_{2}<\cdots<l_{t}, u+\sum_{j=1}^{t} l_{j}=d_{\min }+1, \\
v+\sum_{j=1}^{t}\left(l_{j}-1\right) \geq\left(2 r_{\min }-1\right) d_{\min }
\end{array}
\end{array}\right\} .
$$

Note that if this set was empty for every $t$, then, of course, the conclusion of Theorem 6.5 would hold in all dimensions (this is known to be false in general). If $A_{t} \neq \emptyset$, it is easy to see that

$a(t)=\min \left\{s-k:(k, s) \in A_{t}\right\}=\left(2 r_{\min }-1\right) d_{\min }-\left(d_{\min }+1\right)+t=d(D, \Sigma)+t+1$.

Hence, we obtain that $\min \left\{a(t): t \geq 1, A_{t} \neq \emptyset\right\}=d(D, \Sigma)+2$. Since $\theta_{k, s}^{\infty}$ is an isomorphism for any $(k, s) \notin \bigcup_{t>1} A_{t}$ for each $0 \leq k \leq d_{\text {min }}$, we have the following: 
$(*)_{2}$ If $0 \leq k \leq d_{\min }, \theta_{k, s}^{\infty}$ is an isomorphism for any $(k, s)$ such that $s-k \leq$ $d(D, \Sigma)+1$.

Then, by $(*)_{1}$ and $(*)_{2}$, we know that $\theta_{k, s}^{\infty}: E_{k, s}^{\infty} \stackrel{\cong}{\rightarrow} E_{k, s}^{\infty}$ is an isomorphism for any $(k, s)$ if $s-k \leq d(D, \Sigma)$, and this completes the proof.

Theorem 6.6. For each $D=\left(d_{1}, \cdots, d_{r}\right) \in \mathbb{N}^{r}$ and $D^{\prime}=\left(d_{1}^{\prime}, \cdots, d_{r}^{\prime}\right) \in \mathbb{N}^{r}$ with $D^{\prime}-D \in\left(\mathbb{Z}_{\geq 0}\right)^{r} \backslash\left\{\mathbf{0}_{r}\right\}$, the stabilization map

$$
s_{D, D^{\prime}}: \operatorname{Pol}_{D}^{*}\left(S^{1}, X_{\Sigma}\right) \rightarrow \operatorname{Pol}_{D^{\prime}}^{*}\left(S^{1}, X_{\Sigma}\right)
$$

is a homotopy equivalence through dimension $d(D, \Sigma)$, where the number $d(D, \Sigma)$ is given by $d(D, \Sigma)=2\left(r_{\min }(\Sigma)-1\right) d_{\min }-2$ as in (2.25).

Proof. For each $1 \leq k \leq r$, let us write $m_{k}=d_{k}^{\prime}-d_{k}$. Let $D=D_{0}=$ $\left(d_{1}, \cdots, d_{r}\right)$, and for each $1 \leq k \leq r$ let us write

$$
\begin{aligned}
D_{k} & =D+\sum_{i=1}^{k} m_{i} \boldsymbol{e}_{i}=\left(d_{1}+m_{1}, d_{2}+m_{2}, \cdots, d_{k}+m_{k}, d_{k+1}, d_{k+2}, \cdots, d_{r}\right) \\
& =\left(d_{1}^{\prime}, d_{2}^{\prime}, \cdots, d_{k}^{\prime}, d_{k+1}, d_{k+2}, \cdots, d_{r}\right) .
\end{aligned}
$$

Note that $D_{r}=\left(d_{1}^{\prime}, \cdots, d_{r}^{\prime}\right)=D^{\prime}$ for $k=r$. Now for each $1 \leq i<r$, define the map $f_{i}: \operatorname{Pol}_{D_{i-1}}^{*}\left(S^{1}, X_{\Sigma}\right) \rightarrow \operatorname{Pol}_{D_{i}}^{*}\left(S^{1}, X_{\Sigma}\right)$ by the composite of stabilization maps

$f_{i}=\left(s_{D_{i-1}+\left(m_{i}-1\right) \boldsymbol{e}_{i}, i}\right) \circ\left(s_{D_{i-1}+\left(m_{i}-2\right) \boldsymbol{e}_{i}, i}\right) \circ \cdots \circ\left(s_{D_{i-1}+2 \boldsymbol{e}_{i}, i}\right) \circ\left(s_{D_{i-1}+\boldsymbol{e}_{i}, i}\right) \circ\left(s_{D_{i-1}, i}\right)$.

Since the stabilization map $s_{D_{i-1}+k e_{i}, i}$ is a homotopy equivalence through dimension $d\left(D_{i-1}+k \boldsymbol{e}_{i}, \Sigma\right)$ by Theorem 6.5 and $d\left(D_{i-1}+k \boldsymbol{e}_{i}, \Sigma\right) \leq d\left(D_{i-1}+\right.$ $\left.(k+1) \boldsymbol{e}_{i}, \Sigma\right)$ for each $0 \leq k<r$, the map $f_{i}$ is a homotopy equivalence through dimension $d\left(D_{i-1}, \Sigma\right)$. Furthermore, we can easily see that

$$
s_{D, D^{\prime}}=f_{r-1} \circ f_{r-2} \circ \cdots \circ f_{2} \circ f_{1} \quad \text { (up to homotopy equivalence) }
$$

Since

$$
d(D, \Sigma)=d\left(D_{0}, \Sigma\right) \leq d\left(D_{1}, \Sigma\right) \leq d\left(D_{2}, \Sigma\right) \leq \cdots \leq d\left(D_{r}, \Sigma\right)=d\left(D^{\prime}, \Sigma\right),
$$

the map $s_{D, D^{\prime}}$ is a homotopy equivalence through dimension $d(D, \Sigma)$.

Definition 6.7. Let $D=\left(d_{1}, \cdots, d_{r}\right) \in \mathbb{N}^{r}$ be an $r$-tuple of positive integers. If the condition $\sum_{k=1}^{r} d_{k} \boldsymbol{n}_{k}=\mathbf{0}_{n}$ is satisfied, the map

$$
j_{D}: \operatorname{Pol}_{D}^{*}\left(S^{1}, X_{\Sigma}\right) \rightarrow \Omega \mathcal{Z}_{\mathcal{K}_{\Sigma}}
$$


was already defined in (2.23), and we shall define it when $\sum_{k=1}^{r} d_{k} \boldsymbol{n}_{k} \neq \mathbf{0}_{n}$.

Suppose that $\sum_{k=1}^{r} d_{k} \boldsymbol{n}_{k} \neq \mathbf{0}_{n}$. By the assumption (2.14.1), there is an $r$-tuple $D_{*}=\left(d_{1}^{*}, \cdots, d_{r}^{*}\right) \in \mathbb{N}^{r}$ such that $\sum_{k=1}^{r} d_{k}^{*} \boldsymbol{n}_{k}=\mathbf{0}_{n}$. If we choose a sufficiently large integer $m_{0} \in \mathbb{N}$, then the following condition holds:

$$
d_{k}<m_{0} d_{k}^{*} \quad \text { for each } 1 \leq k \leq r .
$$

Now we set $D_{0}=m_{0} D_{*}=\left(m_{0} d_{1}^{*}, m_{0} d_{2}^{*}, \cdots, m_{0} d_{r}^{*}\right)$ and define the map

$$
j_{D}: \operatorname{Pol}_{D}^{*}\left(S^{1}, X_{\Sigma}\right) \rightarrow \Omega \mathcal{Z}_{\mathcal{K}_{\Sigma}}
$$

as the composite of maps

$$
j_{D}=j_{D_{0}} \circ s_{D, D_{0}}: \operatorname{Pol}_{D}^{*}\left(S^{1}, X_{\Sigma}\right) \stackrel{s_{D, D_{0}}}{\longrightarrow} \operatorname{Pol}_{D_{0}}^{*}\left(S^{1}, X_{\Sigma}\right) \stackrel{j_{D_{0}}}{\longrightarrow} \Omega \mathcal{Z}_{\mathcal{K}_{\Sigma}} \cdot
$$

Similarly, for each $r$-tuple $D=\left(d_{1}, \cdots, d_{r}\right) \in \mathbb{N}^{r}$ of positive integers, define the map

$$
i_{D}: \operatorname{Pol}_{D}^{*}\left(S^{1}, X_{\Sigma}\right) \rightarrow \Omega X_{\Sigma}
$$

as the composite of maps

$$
i_{D}=q_{\Sigma} \circ j_{D}
$$

Remark 6.8. Note that the maps $j_{D}$ and $i_{D}$ depend on the choice of the pair $\left(D_{*}, m_{0}\right)$, but one can show that their homotopy classes do not depend on this pair.

\section{Scanning maps}

In this section we consider configuration spaces and the scanning map. In particular, we recall the definition of the horizontal scanning map and prove that it is a homotopy equivalence.

Definition 7.1. For a positive integer $d \geq 1$ and a based space $X$, let $\operatorname{SP}^{d}(X)$ denote the $d$-th symmetric product of $X$ defined as the orbit space

$$
\mathrm{SP}^{d}(X)=X^{d} / S_{d}
$$

where the symmetric group $S_{d}$ of $d$ letters acts on the $d$-fold product $X^{d}$ in the natural manner. 
Remark 7.2. (i) Note that an element $\eta \in \mathrm{SP}^{d}(X)$ may be identified with a formal linear combination

$$
\eta=\sum_{k=1}^{s} n_{k} x_{k}
$$

where $\left\{x_{k}\right\}_{k=1}^{s} \in C_{s}(X)$ and $\left\{n_{k}\right\}_{k=1}^{s} \subset \mathbb{N}$ with $\sum_{k=1}^{s} n_{k}=d$.

(ii) For example, when $X=\mathbb{C}$, we have the natural homeomorphism

$$
\psi_{d}: \mathrm{P}^{d}(\mathbb{C}) \stackrel{\cong}{\longrightarrow} \mathrm{SP}^{d}(\mathbb{C})
$$

given by using the above identification

$$
\psi_{d}(f(z))=\psi_{d}\left(\prod_{k=1}^{s}\left(z-\alpha_{k}\right)^{d_{k}}\right)=\sum_{k=1}^{s} d_{k} \alpha_{k}
$$

for $f(z)=\prod_{k=1}^{s}\left(z-\alpha_{k}\right)^{d_{k}} \in \mathrm{P}^{d}(\mathbb{C})$.

Definition 7.3. (i) For a subspace $A \subset X$, let $\operatorname{SP}^{d}(X, A)$ denote the quotient space

$$
\mathrm{SP}^{d}(X, A)=\mathrm{SP}^{d}(X) / \sim
$$

where the equivalence relation $\sim$ is defined by

$$
\xi \sim \eta \Leftrightarrow \xi \cap(X \backslash A)=\eta \cap(X \backslash A) \quad \text { for } \xi, \eta \in \mathrm{SP}^{d}(X) .
$$

Thus, the points of $A$ are ignored. When $A \neq \emptyset$, by adding a point in $A$ we have the natural inclusion

$$
\mathrm{SP}^{d}(X, A) \subset \mathrm{SP}^{d+1}(X, A) .
$$

Thus, when $A \neq \emptyset$, one can define the space $\operatorname{SP}^{\infty}(X, A)$ by the union

$$
\mathrm{SP}^{\infty}(X, A)=\bigcup_{d \geq 0} \mathrm{SP}^{d}(X, A)
$$

where we set $\operatorname{SP}^{0}(X, A)=\{\emptyset\}$ and $\emptyset$ denotes the empty configuration.

(ii) From now on, we always assume that $X \subset \mathbb{C}$. For each $r$-tuple $D=\left(d_{1}, \cdots, d_{r}\right) \in \mathbb{N}^{r}$, let $\mathrm{SP}^{D}(X)=\prod_{i=1}^{r} \operatorname{SP}^{d_{i}}(X)$, and define the spaces $E_{D}^{\Sigma, \mathbb{R}}(X)$ and $E_{D}^{\Sigma}(X)$ by

$$
\begin{gathered}
E_{D}^{\Sigma, \mathbb{R}}(X)=\left\{\left(\xi_{1}, \cdots, \xi_{r}\right) \in \operatorname{SP}^{D}(X):\left(\bigcap_{k \in \sigma} \xi_{k}\right) \cap \mathbb{R}=\emptyset \text { for any } \sigma \in I\left(\mathcal{K}_{\Sigma}\right)\right\} \\
E_{D}^{\Sigma}(X)=\left\{\left(\xi_{1}, \cdots, \xi_{r}\right) \in \operatorname{SP}^{D}(X): \bigcap_{k \in \sigma} \xi_{k}=\emptyset \text { for any } \sigma \in I\left(\mathcal{K}_{\Sigma}\right)\right\} .
\end{gathered}
$$


(iii) When $A \subset X$ is a subspace, define an equivalence relation " $~$ " on the space $E_{D}^{\Sigma, \mathbb{R}}(X)$ (resp. the space $\left.E_{D}^{\Sigma}(X)\right)$ by

$\left(\xi_{1}, \cdots, \xi_{r}\right) \sim\left(\eta_{1}, \cdots, \eta_{r}\right) \quad$ if $\quad \xi_{i} \cap(X \backslash A)=\eta_{i} \cap(X \backslash A) \quad$ for each $1 \leq j \leq r$.

Let $E_{D}^{\Sigma, \mathbb{R}}(X, A)$ and $E_{D}^{\Sigma}(X, A)$ be the quotient spaces defined by

$$
E_{D}^{\Sigma, \mathbb{R}}(X, A)=E_{D}^{\Sigma, \mathbb{R}}(X) / \sim \text {, and } E_{D}^{\Sigma}(X, A)=E_{D}^{\Sigma}(X) / \sim .
$$

When $A \neq \emptyset$, by adding points in $A$ we have natural inclusions

$$
E_{D}^{\Sigma, \mathbb{R}}(X, A) \subset E_{D+e_{i}}^{\Sigma, \mathbb{R}}(X, A), \quad E_{D}^{\Sigma}(X, A) \subset E_{D+e_{i}}^{\Sigma}(X, A)
$$

for each $1 \leq i \leq r$, where $D+\boldsymbol{e}_{i}=\left(d_{1}, \cdots, d_{i-1}, d_{i}+1, d_{i+1}, \cdots, d_{r}\right)$ as in $(5.15)$. Thus, when $A \neq \emptyset$, one can define the spaces $E^{\Sigma, \mathbb{R}}(X, A)$ and $E^{\Sigma}(X, A)$ by the unions

$$
E^{\Sigma, \mathbb{R}}(X, A)=\bigcup_{D \in \mathbb{N}^{r}} E_{D}^{\Sigma, \mathbb{R}}(X, A), \quad E^{\Sigma}(X, A)=\bigcup_{D \in \mathbb{N}^{r}} E_{D}^{\Sigma}(X, A),
$$

where the empty configuration $(\emptyset, \cdots, \emptyset)$ is the base-point of $E^{\Sigma, \mathbb{R}}(X, A)$ or $E^{\Sigma}(X, A)$.

Remark 7.4. (i) If $X \subset \mathbb{R}$, the following equalities hold:

$$
E_{D}^{\Sigma, \mathbb{R}}(X)=E_{D}^{\Sigma}(X) \quad \text { and } \quad E^{\Sigma, \mathbb{R}}(X, A)=E^{\Sigma}(X, A) .
$$

(ii) Let $D=\left(d_{1}, \cdots, d_{r}\right) \in \mathbb{N}^{r}$. Then by using the identification (7.3) we easily obtain the homeomorphism

$$
\begin{aligned}
& \operatorname{Pol}_{D}^{*}\left(S^{1}, X_{\Sigma}\right) \quad \stackrel{\Psi_{D}}{\cong} \quad E_{D}^{\Sigma, \mathbb{R}}(\mathbb{C}) \\
& \left(f_{1}(z), \cdots, f_{r}(z)\right) \longrightarrow\left(\psi_{d_{1}}\left(f_{1}(z)\right), \cdots, \psi_{d_{r}}\left(f_{r}(z)\right)\right)
\end{aligned}
$$

(iii) Now let $\varphi_{D}: \mathbb{C} \cong U_{D}$ and $\boldsymbol{x}_{D}=\left(x_{D, 1}, \cdots, x_{D, r}\right) \in F\left(\mathbb{C} \backslash \overline{U_{D}}, r\right)$ be the homeomorphism and the point for defining the stabilization map $s_{D}$ in Definition 6.1. Then define the map

$$
\begin{gathered}
s_{D}^{\Sigma}: E_{D}^{\Sigma, \mathbb{R}}(\mathbb{C}) \rightarrow E_{D+e}^{\Sigma, \mathbb{R}}(\mathbb{C}) \quad \text { by } \\
s_{D}^{\Sigma}\left(\xi_{1}, \cdots, \xi_{r}\right)=\left(\varphi_{D}\left(\xi_{1}\right)+x_{D, 1}, \cdots, \varphi_{D}\left(\xi_{r}\right)+x_{D, r}\right)
\end{gathered}
$$

for $\left(\xi_{1}, \cdots, \xi_{r}\right) \in E_{D}^{\Sigma, \mathbb{R}}$, where we write $\varphi_{D}(\xi)=\sum_{k=1}^{s} n_{k} \varphi_{D}\left(x_{k}\right)$ if $\xi=$ $\sum_{k=1}^{s} n_{k} x_{k} \in \mathrm{SP}^{d}(\mathbb{C})$ and $\left(n_{k}, x_{k}\right) \in \mathbb{N} \times \mathbb{C}$ with $\sum_{k=1}^{s} n_{k}=d$. 
Then by using the above homeomorphism (7.8), we have the following commutative diagram

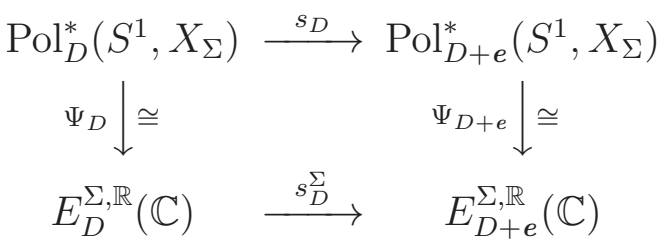

(iv) Note that $E_{D}^{\Sigma, \mathbb{R}}(\mathbb{C})$ is path-connected. Indeed, for any two points $\xi_{0}, \xi_{1} \in E_{D}^{\Sigma}(\mathbb{C})$, one can construct a path $\omega:[0,1] \rightarrow E_{D}^{\Sigma, \mathbb{R}}(\mathbb{C})$ such that $\omega(i)=\xi_{i}$ for $i \in\{0,1\}$ by using the string representation used in [12, $\S$ Appendix]. Thus the space $\operatorname{Pol}_{D}^{*}\left(S^{1}, X_{\Sigma}\right)$ is also path-connected.

Definition 7.5. Define the stabilized space $\mathrm{Pol}_{D+\infty}^{\Sigma}$ by the colimit

$$
\operatorname{Pol}_{D+\infty}^{\Sigma}=\lim _{k \rightarrow \infty} \operatorname{Pol}_{D+k e}^{*}\left(S^{1}, X_{\Sigma}\right)
$$

where the colimt is taken from the family of stabilization maps

$$
\left\{s_{D+k e}: \operatorname{Pol}_{D+k e}^{*}\left(S^{1}, X_{\Sigma}\right) \rightarrow \operatorname{Pol}_{D+(k+1) e}^{*}\left(S^{1}, X_{\Sigma}\right)\right\}_{k \geq 0}
$$

Now we are ready to define the scanning map. From now on, we identify $\mathbb{C}=\mathbb{R}^{2}$ in a usual way.

Definition 7.6. For a rectangle $X$ in $\mathbb{C}=\mathbb{R}^{2}$, let $\sigma X$ denote the union of the sides of $X$ which are parallel to the $y$-axis, and for a subspace $Z \subset \mathbb{C}=\mathbb{R}^{2}$, let $\bar{Z}$ be the closure of $Z$. From now on, let $I$ denote the interval $I=[-1,1]$ and let $0<\epsilon<\frac{1}{1000}$ be any fixed real number.

For each $x \in \mathbb{R}$, let $V(x)$ be the set defined by

$$
\begin{aligned}
V(x) & =\{w \in \mathbb{C}:|\operatorname{Re}(w)-x|<\epsilon,|\operatorname{Im}(w)|<1\} \\
& =(x-\epsilon, x+\epsilon) \times(-1,1),
\end{aligned}
$$

and let's identify $I \times I=I^{2}$ with the closed unit rectangle $\{t+s \sqrt{-1} \in \mathbb{C}$ : $-1 \leq t, s \leq 1\}$ in $\mathbb{C}$.

For each $D=\left(d_{1}, \cdots, d_{r}\right) \in \mathbb{N}^{r}$, define the horizontal scanning map

$$
s c_{D}: E_{D}^{\Sigma, \mathbb{R}}(\mathbb{C}) \rightarrow \Omega E^{\Sigma, \mathbb{R}}\left(I^{2}, \partial I \times I\right)=\Omega E^{\Sigma, \mathbb{R}}\left(I^{2}, \sigma I^{2}\right)
$$

as follows. For each $r$-tuple $\alpha=\left(\xi_{1}, \cdots, \xi_{r}\right) \in E_{D}^{\Sigma, \mathbb{R}}(\mathbb{C})$ of configurations, let $s c_{D}(\alpha): \mathbb{R} \rightarrow E^{\Sigma, \mathbb{R}}\left(I^{2}, \partial I \times I\right)=E^{\Sigma, \mathbb{R}}\left(I^{2}, \sigma I^{2}\right)$ denote the map given by $\mathbb{R} \ni x \mapsto\left(\xi_{1} \cap \bar{V}(x), \cdots, \xi_{r} \cap \bar{V}(x)\right) \in E^{\Sigma, \mathbb{R}}(\bar{V}(x), \sigma \bar{V}(x)) \cong E^{\Sigma, \mathbb{R}}\left(I^{2}, \sigma I^{2}\right)$, 
where we use the canonical identification $(\bar{V}(x), \sigma \bar{V}(x)) \cong\left(I^{2}, \sigma I^{2}\right)$.

Since $\lim _{x \rightarrow \pm \infty} s c_{D}(\alpha)(x)=(\emptyset, \cdots, \emptyset)$, by setting $s c_{D}(\alpha)(\infty)=(\emptyset, \cdots, \emptyset)$ we obtain a based map $s c_{D}(\alpha) \in \Omega E^{\Sigma, \mathbb{R}}\left(I^{2}, \sigma I^{2}\right)$, where we identify $S^{1}=$ $\mathbb{R} \cup \infty$ and we choose the empty configuration $(\emptyset, \cdots, \emptyset)$ as the base-point of $E^{\Sigma, \mathbb{R}}\left(I^{2}, \sigma I^{2}\right)$. One can show that the following diagram is homotopy commutative:

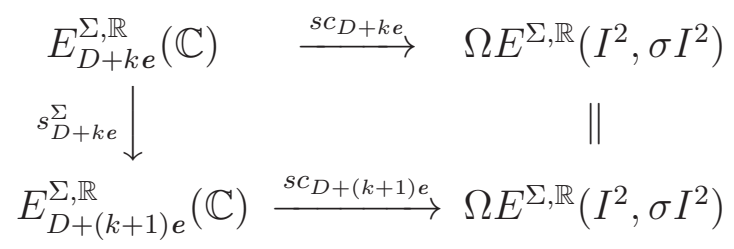

Thus by using the above diagram and by identifying $\operatorname{Pol}_{D+k e}^{*}\left(S^{1}, X_{\Sigma}\right)$ with $E_{D+k e}^{\Sigma, \mathbb{R}}(\mathbb{C})$, we finally obtain the stable horizontal scanning map

$$
S=\lim _{k \rightarrow \infty} s c_{D+k e}: \operatorname{Pol}_{D+\infty}^{\Sigma} \rightarrow \Omega E^{\Sigma, \mathbb{R}}\left(I^{2}, \sigma I^{2}\right),
$$

where $\operatorname{Pol}_{D+\infty}^{\Sigma}$ is defined in (7.11).

(ii) If we identify $(I, \partial I)=(I \times\{0\}, \partial I \times\{0\})$, then we notice that $\left(\xi_{1} \cap \mathbb{R}, \cdots, \xi_{r} \cap \mathbb{R}\right) \in E^{\Sigma, \mathbb{R}}(I, \partial I)$ for $\left(\xi_{1}, \cdots, \xi_{r}\right) \in E^{\Sigma, \mathbb{R}}\left(I^{2}, \sigma I^{2}\right)$. Thus one can define the map $R_{\Sigma}: E^{\Sigma, \mathbb{R}}\left(I^{2}, \sigma I^{2}\right) \rightarrow E^{\Sigma, \mathbb{R}}(I, \partial I)$ by

$$
R_{\Sigma}\left(\xi_{1}, \cdots, \xi_{r}\right)=\left(\xi_{1} \cap \mathbb{R}, \cdots, \xi_{r} \cap \mathbb{R}\right) \text { for }\left(\xi_{1}, \cdots, \xi_{r}\right) \in E^{\Sigma, \mathbb{R}}\left(I^{2}, \sigma I^{2}\right) \text {. }
$$

(iii) Let $S^{H}: \mathrm{Pol}_{D+\infty}^{\Sigma} \rightarrow \Omega E^{\Sigma, \mathbb{R}}(I, \partial I)$ denote the horizontal scanning map defined by the composite of maps

$$
S^{H}=\left(\Omega R_{\Sigma}\right) \circ S: \mathrm{Pol}_{D+\infty}^{\Sigma} \stackrel{S}{\longrightarrow} \Omega E^{\Sigma, \mathbb{R}}\left(I^{2}, \sigma I^{2}\right) \stackrel{\Omega R_{\Sigma}}{\longrightarrow} \Omega E^{\Sigma, \mathbb{R}}(I, \partial I),
$$

where $S$ denotes the stable horizontal scanning map given by (7.15).

Lemma 7.7. The map $R_{\Sigma}: E^{\Sigma, \mathbb{R}}\left(I^{2}, \sigma I^{2}\right) \stackrel{\simeq}{\longrightarrow} E^{\Sigma, \mathbb{R}}(I, \partial I)=E^{\Sigma}(I, \partial I)$ is a deformation retraction.

Proof. We identify $I^{2}=\{a+b \sqrt{-1} \in \mathbb{C}:-1 \leq a, b \leq 1\} \subset \mathbb{C}$ as before. Let $\Pi \subset I^{2}$ denote the subspace defined by $\Pi=\left\{a+b \sqrt{-1} \in I^{2}: b \in\left\{0, \pm \frac{1}{2}\right\}\right\}$. For $b \in \mathbb{R}$, let $\epsilon(b)=\frac{b}{|b|}$ if $b \neq 0$ and $\epsilon(0)=0$. Now consider the homotopy $\varphi: I^{2} \times[0,1] \rightarrow I^{2}$ given by $\varphi(\alpha, t)=a+\left\{(1-t) b+\frac{\epsilon(b) t}{2}\right\} \sqrt{-1}$ for $\alpha=$ $a+b \sqrt{-1} \in I^{2}(a, b \in \mathbb{R})$. By means of this homotopy, one can define a deformation retraction $R: E^{\Sigma, \mathbb{R}}\left(I^{2}, \sigma I^{2}\right) \stackrel{\simeq}{\longrightarrow} E^{\Sigma, \mathbb{R}}\left(\Pi, \partial I \times\left\{0, \pm \frac{1}{2}\right\}\right)$.

Next, by using the homotopy given by $f_{t}(a+b \sqrt{-1})=t a+(1-t)+b \sqrt{-1}$ if $b= \pm \frac{1}{2}$ and $f_{t}(a+b \sqrt{-1})=a$ if $b=0$, one can also define a deformation retraction $\varphi: E^{\Sigma, \mathbb{R}}\left(\Pi, \partial I \times\left\{0, \pm \frac{1}{2}\right\}\right) \stackrel{\simeq}{\longrightarrow} E^{\Sigma, \mathbb{R}}(I, \partial I)$. Since $R_{\Sigma}=\varphi \circ R$, the map $R_{\Sigma}$ is a deformation retraction. 
Theorem 7.8 ([10], [26]). The horizontal scanning map

$$
S^{H}: \mathrm{Pol}_{D+\infty}^{\Sigma} \stackrel{\simeq}{\longrightarrow} \Omega E^{\Sigma}(I, \partial I)
$$

is a homotopy equivalence.

Proof. Since $R_{\Sigma}$ is a homotopy equivalence and $S^{H}=\left(\Omega R_{\Sigma}\right) \circ S$, it suffices to show that $S$ is a homotopy equivalence.

The proof is analogous to the one given in [26, Prop. 3.2, Lemma 3.4] and [10, Prop. 2]. We identify $\mathbb{C}=\mathbb{R}^{2}$ by means of the identification $x+\sqrt{-1} y \mapsto$ $(x, y)$ in the usual way. Let $B$ and $B^{*}$ denote the rectangles in $\mathbb{R}^{2}=\mathbb{C}$ given by $B^{*}=[-1,2] \times[-1,1]$ and $B=(0,1) \times(-1,1)$. Let $\left\{V_{t}: 0<t<1\right\}$ be the family of open rectangles in $B$ given by $V_{t}=(t-\epsilon(t), t+\epsilon(t)) \times(-1,1)$, where $\epsilon(t)$ denotes the continuous function defined on the open interval $(0,1)$ such that $0<\epsilon(t)<\min \{t, 1-t\}$ for any $t \in(0,1)$ with $\lim _{t \rightarrow+0} \epsilon(t)=\lim _{t \rightarrow 1-0} \epsilon(t)=0$.

Let $\widetilde{s c}_{D}^{H}: E_{D}^{\Sigma, \mathbb{R}}(B) \times[0,1] \rightarrow E^{\Sigma, \mathbb{R}}(\bar{B}, \sigma \bar{B})$ denote the map given by

$$
\widetilde{s c}_{D}^{H}\left(\left(\xi_{1}, \cdots, \xi_{r}\right), t\right)=\left(\xi_{1} \cap V_{t}, \cdots, \xi_{r} \cap V_{t}\right) \in E^{\Sigma, \mathbb{R}}\left(\overline{V_{t}}, \sigma \overline{V_{t}}\right) \cong E^{\Sigma, \mathbb{R}}(\bar{B}, \sigma \bar{B}),
$$

where we use the canonical identification $\left(\overline{V_{t}}, \sigma \overline{V_{t}}\right) \cong(\bar{B}, \sigma \bar{B})$.

Since $\lim _{t \rightarrow+0} \widetilde{s c}_{D}^{H}(\xi, t)=\lim _{t \rightarrow 1-0} \widetilde{s c}_{D}^{H}(\xi, t)=(\emptyset, \cdots, \emptyset)$ for any $\xi \in E_{D}^{\Sigma, \mathbb{R}}(B)$, the adjoint of $\widetilde{s c}_{D}^{H}$ defines the map $s c_{D}^{H}: E_{D}^{\Sigma, \mathbb{R}}(B) \rightarrow \Omega E^{\Sigma, \mathbb{R}}(\bar{B}, \sigma \bar{B})$.

If $s_{D}^{\Sigma}: E_{D}^{\Sigma, \mathbb{R}}(B) \rightarrow E_{D+e}^{\Sigma, \mathbb{R}}(B)$ denotes the stabilization map defined by adding points from infinity as in (7.10), we obtain a homotopy commutative diagram

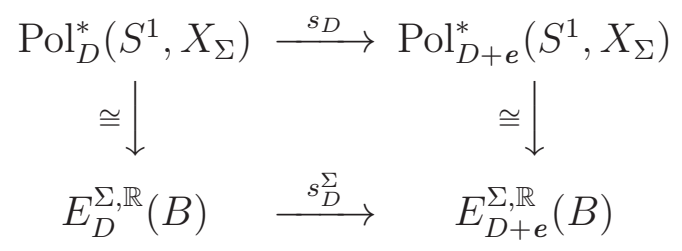

for each $D \in \mathbb{N}^{r}$. If $E_{D+\infty}^{\Sigma, \mathbb{R}}(B)$ denotes the colimit $E_{D+\infty}^{\Sigma, \mathbb{R}}(B)=\lim _{k \rightarrow \infty} E_{D+k e}^{\Sigma, \mathbb{R}}(B)$ taken over the stabilization maps $\left\{s_{D,+k e}^{\Sigma}: E_{D+k e}^{\Sigma, \mathbb{R}}(B) \rightarrow E_{D+(k+1) e}^{\Sigma, \mathbb{R}}(B)\right\}$, there is a homotopy commutative diagram

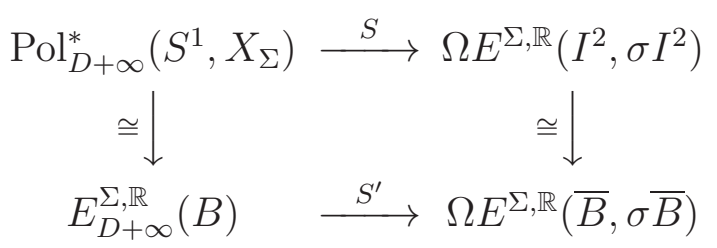

where we set $S^{\prime}=\lim _{k \rightarrow \infty} s c_{D+k e}^{H}$. It suffices to prove that $S^{\prime}$ is a homotopy equivalence. Let $E_{D}^{\Sigma, \mathbb{R}}$ denote the subspace of $E^{\Sigma, \mathbb{R}}\left(B^{*}, \sigma B^{*}\right)$ consisting of 
all $r$-tuples $\left(\xi_{1}, \cdots, \xi_{r}\right) \in E^{\Sigma, \mathbb{R}}\left(B^{*}, \sigma B^{*}\right)$ such that $\left(\xi_{1} \cap B, \cdots, \xi_{r} \cap B\right) \in$ $E_{D}^{\Sigma, \mathbb{R}}(B)$.

Let $q_{D}: E_{D}^{\Sigma, \mathbb{R}} \rightarrow E^{\Sigma, \mathbb{R}}\left(B^{*}, \sigma B^{*} \cup B\right) \cong E^{\Sigma, \mathbb{R}}(\bar{B}, \sigma \bar{B})^{2}$ denote the restriction of the the quotient map $E^{\Sigma, \mathbb{R}}\left(B^{*}, \sigma B^{*}\right) \rightarrow E^{\Sigma, \mathbb{R}}\left(B^{*}, \sigma B^{*} \cup B\right) \cong$ $E^{\Sigma, \mathbb{R}}(\bar{B}, \sigma \bar{B})^{2}$, where $E^{\Sigma, \mathbb{R}}(\bar{B}, \sigma \bar{B})^{2}=E^{\Sigma, \mathbb{R}}(\bar{B}, \sigma \bar{B}) \times E^{\Sigma, \mathbb{R}}(\bar{B}, \sigma \bar{B})$. It is easy to see that the fiber of $q_{D}$ is homeomorphic to $E_{D}^{\Sigma, \mathbb{R}}(B)$.

Next, define the scanning map $\tilde{S}_{D}: E_{D}^{\Sigma, \mathbb{R}} \rightarrow \operatorname{Map}\left([0,1], E^{\Sigma, \mathbb{R}}(\bar{B}, \sigma \bar{B})\right)$ by

$$
\begin{gathered}
E_{D}^{\Sigma, \mathbb{R}} \times[0,1] \longrightarrow E^{\Sigma, \mathbb{R}}(\bar{B}, \sigma \bar{B}) \\
\left(\left(\xi_{1}, \cdots, \xi_{r}\right), t\right) \longrightarrow\left(\xi_{1} \cap B_{t}, \cdots, \xi_{r} \cap B_{t}\right) \in E^{\Sigma, \mathbb{R}}\left(\overline{B_{t}}, \sigma \overline{B_{t}}\right) \cong E^{\Sigma, \mathbb{R}}(\bar{B}, \sigma \bar{B})
\end{gathered}
$$

where $\left\{B_{t}: 0<t<1\right\}$ denotes the family of the open rectangles in $B^{*}$ defined by $B_{t}=(2 t-1,2 t) \times(-1,1)$ and we use the canonical identification $\left(\overline{B_{t}}, \sigma \overline{B_{t}}\right) \cong(\bar{B}, \sigma \bar{B})$. We obtain a homotopy commutative diagram

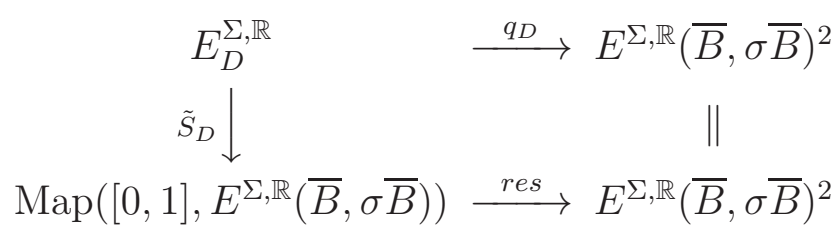

where

res : $\operatorname{Map}\left([0,1], E^{\Sigma, \mathbb{R}}(\bar{B}, \sigma \bar{B})\right) \rightarrow \operatorname{Map}\left(\{0,1\}, E^{\Sigma, \mathbb{R}}(\bar{B}, \sigma \bar{B})\right) \cong E^{\Sigma, \mathbb{R}}(\bar{B}, \sigma \bar{B})^{2}$

denotes the restriction map.

Note that res is a fibration with fibre $\Omega E^{\Sigma, \mathbb{R}}(\bar{B}, \sigma \bar{B})$, but the map $q_{D}$ is not a fibration although every fiber of $q_{D}$ is homeomorphic to $E^{\Sigma, \mathbb{R}}(B)$. However, once the map $q_{D}$ is stabilized, it becomes a quasifibration.

To see this, let $J=(0,1)$ and for each $D \in \mathbb{N}^{r}$ let us choose the $r$-tuple $\boldsymbol{c}_{D}=\left(c_{D, 1}, \cdots, c_{D, r}\right) \in(J \times J)^{r}$ such that $c_{D, j} \neq c_{D^{\prime}, k}$ if $(D, j) \neq\left(D^{\prime}, k\right)$ with $\lim _{D \rightarrow \infty} c_{D, k}=\left(\frac{1}{2}, 0\right)$ for each $1 \leq k \leq r$.

Let $\widehat{E^{\Sigma, \mathbb{R}}}\left(B^{*}, \sigma B^{*}\right)$ denote the space of $r$-tuples $\left(\xi_{1}, \cdots, \xi_{r}\right)$ of formal infinite divisors in $\left(B^{*}, \sigma B^{*}\right)$ satisfying the following two conditions:

$(\dagger)_{1}\left(\bigcap_{k=1}^{r} \xi_{k}\right) \cap \mathbb{R} \cap\left(B^{*} \backslash \sigma B^{*}\right)=\emptyset$.

$(\dagger)_{2}$ Each divisor $\xi_{k}$ is represented as the formal infinite sum of the form $\xi_{k}=\sum_{D} \xi_{k, D}$ such that $\xi_{k, D} \in \mathrm{SP}^{1}\left(B^{*}, \sigma B^{*}\right)$ for each $D \in \mathbb{N}^{r}$, and it almost coincides (except finite sums) with $\xi_{k}^{*}=\sum_{D} c_{D, k}$ for each $1 \leq k \leq r$. 
Note that $c_{D, k} \in B$ for any $(D, k) \in \mathbb{N}^{r} \times[r]$. Thus one can define the map $\hat{q}: \widehat{E^{\Sigma, \mathbb{R}}}\left(B^{*}, \sigma B^{*}\right) \rightarrow E^{\Sigma, \mathbb{R}}(\bar{B}, \sigma \bar{B})^{2}$ to be the natural quotient map

$$
\widehat{E^{\Sigma, \mathbb{R}}}\left(B^{*}, \sigma B^{*}\right) \rightarrow E^{\Sigma, \mathbb{R}}\left(B^{*}, \sigma B^{*} \cup B\right) \cong E^{\Sigma, \mathbb{R}}(\bar{B}, \sigma \bar{B})^{2} .
$$

By using the Dold-Thom argument exactly as in [26, Lemma 3.4] together with the fact that $E_{D}^{\Sigma, \mathbb{R}}(\mathbb{C})$ is simply connected (by Proposition 5.9 and (7.8)), one can show that $\hat{q}$ is a quasifibration.

Now define stabilization maps $f_{D}: E_{D}^{\Sigma, \mathbb{R}} \rightarrow E_{D+e}^{\Sigma, \mathbb{R}}$ by $f_{D}\left(\xi_{1}, \cdots, \xi_{r}\right)=$ $\left(\xi_{1}+c_{D, 1}, \cdots, \xi_{r}+c_{D, r}\right)$ for $\left(\xi_{1}, \cdots, \xi_{r}\right) \in E_{D}^{\Sigma, \mathbb{R}}$.

Let $E^{\Sigma, \mathbb{R}}$ denote the colimit $E^{\Sigma, \mathbb{R}}=\lim _{k \rightarrow \infty} E_{D+k e}^{\Sigma, \mathbb{R}}$ from the stabilization maps $\left\{f_{D+k e}\right\}_{k \geq 0}$. Since $\widehat{E^{\Sigma, \mathbb{R}}}\left(B^{*}, \sigma B^{*}\right)$ is $\mathbb{Z}^{r} \times E^{\Sigma, \mathbb{R}}$, the restriction $q_{\infty}=$ $\hat{q} \mid E^{\Sigma, \mathbb{R}}: E^{\Sigma, \mathbb{R}} \rightarrow E^{\Sigma, \mathbb{R}}(\bar{B}, \sigma \bar{B})^{2}$ is also a quasifibration. Since the fiber of $q_{D}$ is $E^{\Sigma, \mathbb{R}}(B)$ and $q_{\infty} \mid E^{\Sigma, \mathbb{R}}=q_{D}$, we see that the fiber of $q_{\infty}$ is $E^{\Sigma, \mathbb{R}}(B)$ and we may regard the map $q_{\infty}$ as the stabilized map of $\left\{q_{D+k e}\right\}_{k \geq 0}$.

We also obtain the stabilized scanning map

$$
\tilde{S}=\lim _{k \rightarrow \infty} \tilde{S}_{D+k e}: E^{\Sigma, \mathbb{R}}=\lim _{k \rightarrow \infty} E_{D+k e}^{\Sigma, \mathbb{R}} \rightarrow \operatorname{Map}\left([0,1], E^{\Sigma, \mathbb{R}}(\bar{B}, \sigma \bar{B})\right) .
$$

Since $[0,1]$ is contractible and there is a homotopy equivalence $E^{\Sigma, \mathbb{R}}=$ $\lim _{k \rightarrow \infty} E_{D+k \boldsymbol{e}}^{\Sigma, \mathbb{R}} \simeq E^{\Sigma, \mathbb{R}}(\bar{B}, \sigma \bar{B})$, we see that $\tilde{S}$ is a homotopy equivalence. Since $B \subset B^{*} \backslash \sigma B^{*}$, we may regard $E^{\Sigma, \mathbb{R}}(B)$ as a subspace of $E^{\Sigma, \mathbb{R}}\left(B^{*}, \sigma B^{*}\right)$ and we see that $\tilde{S}_{D} \mid E^{\Sigma, \mathbb{R}}(B)=s c_{D}^{H}$. Thus, we can identify $\tilde{S} \mid E^{\Sigma, \mathbb{R}}(B)=S^{\prime}$ and by the diagram (7.18) we have the homotopy commutative diagram

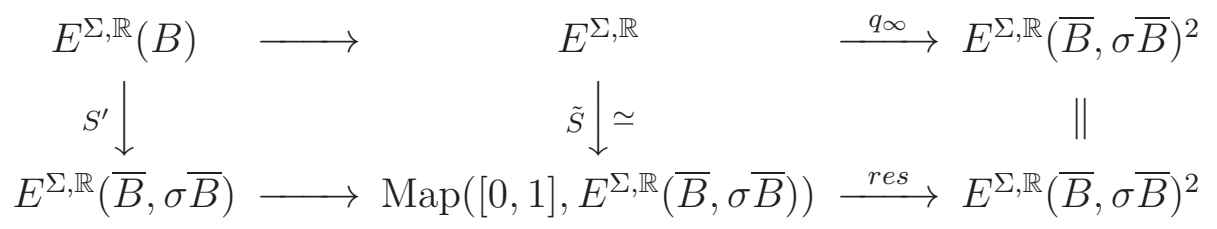

Since the upper horizontal sequence is a quasifibration sequence and the lower horizontal one is a fibration sequence, $S^{\prime}$ is a homotopy equivalence.

\section{The stable result}

In this section we prove the stability result (Theorem 8.2).

Definition 8.1. Let $D=\left(d_{1}, \cdots, d_{r}\right) \in \mathbb{N}^{r}$ be an $r$-tuple of positive integers. 
Then it is easy to see that the following diagram is homotopy commutative:

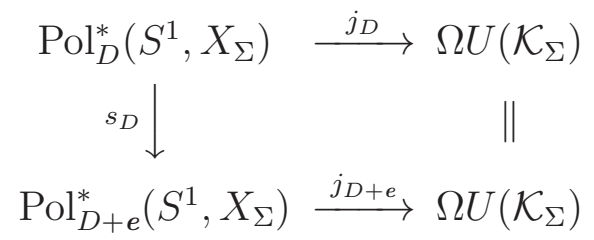

Hence we can stabilize the map

$$
j_{D+\infty}=\lim _{t \rightarrow \infty} j_{D+t e}: \operatorname{Pol}_{D+\infty}^{\Sigma}=\lim _{t \rightarrow \infty} \operatorname{Pol}_{D+t e}^{*}\left(S^{1}, X_{\Sigma}\right) \rightarrow \Omega U\left(\mathcal{K}_{\Sigma}\right) .
$$

The main purpose of this section is to prove the following result.

Theorem 8.2. The map $j_{D+\infty}: \operatorname{Pol}_{D+\infty}^{\Sigma} \stackrel{\simeq}{\longrightarrow} \Omega U\left(\mathcal{K}_{\Sigma}\right)$ is a homotopy equivalence.

Before proving Theorem 8.2 we need the following definition and lemma.

Definition 8.3. Now we identify $\mathbb{C}=\mathbb{R}^{2}$ in a usual way and let us write $U=\{w \in \mathbb{C}:|\operatorname{Re}(w)|<1,|\operatorname{Im}(w)|<1\}=(-1,1) \times(-1,1)$ and $I=[-1,1]$.

(i) For an open set $X \subset \mathbb{C}$, let $F(X)$ denote the space of $r$-tuples $\left(f_{1}(z), \cdots, f_{r}(z)\right) \in \mathbb{C}[z]^{r}$ of (not necessarily monic) polynomials satisfying the following condition $(*)$ :

$(*)$ For any $\sigma=\left\{i_{1}, \cdots, i_{s}\right\} \in I\left(\mathcal{K}_{\Sigma}\right)$, the polynomials $f_{i_{1}}(z), \cdots, f_{i_{s}}(z)$ have no common real roots in $X$ (i.e. no common roots in $X \cap \mathbb{R}$ ).

Similarly, let $F^{\mathbb{C}}(X) \subset F(X)$ denote the subspace of all $\left(f_{1}(z), \cdots, f_{r}(z)\right) \in$ $F(X)$ satisfying the following condition $(*)_{\mathbb{C}}$ :

$(*)_{\mathbb{C}}$ For any $\sigma=\left\{i_{1}, \cdots, i_{s}\right\} \in I\left(\mathcal{K}_{\Sigma}\right)$, the polynomials $f_{i_{1}}(z), \cdots, f_{i_{s}}(z)$ have no common roots in $X$.

(ii) Let $e v_{0}: F(U) \rightarrow U\left(\mathcal{K}_{\Sigma}\right)$ denote the map given by evaluation at 0 , i.e. $e v_{0}\left(f_{1}(z), \cdots, f_{r}(z)\right)=\left(f_{1}(0), \cdots, f_{r}(0)\right)$ for $\left(f_{1}(z), \cdots, f_{r}(z)\right) \in F(U)$.

(iii) Let $\tilde{F}(U) \subset F(U)$ (resp. $\tilde{F}^{\mathbb{C}}(U) \subset F^{\mathbb{C}}(U)$ ) denote the subspace of all $\left(f_{1}(z), \cdots, f_{r}(z)\right) \in F(U)\left(\operatorname{resp} . \quad\left(f_{1}(z), \cdots, f_{r}(z)\right) \in F^{\mathbb{C}}(U)\right)$ such that no $f_{i}(z)$ is identically zero. Let $j_{F}: \tilde{F}^{\mathbb{C}}(U) \stackrel{\subset}{\longrightarrow} \tilde{F}(U)$ denote the inclusion map.

(iv) Let $e v_{\mathbb{R}}: \tilde{F}(U) \rightarrow U\left(\mathcal{K}_{\Sigma}\right)$ denote the map given by the restriction $e v_{\mathbb{R}}=e v_{0} \mid \tilde{F}(U)$.

(v) Note that the group $\mathbb{T}_{\mathbb{R}}^{r}=\left(\mathbb{R}^{*}\right)^{r}$ (resp. $\left.\mathbb{T}_{\mathbb{C}}^{r}=\left(\mathbb{C}^{*}\right)^{r}\right)$ acts freely on the space $\tilde{F}(U)$ (resp. $\left.\tilde{F}^{\mathbb{C}}(U)\right)$ in a natural way, and let

$$
p: \tilde{F}(U) \rightarrow \tilde{F}(U) / \mathbb{T}_{\mathbb{R}}^{r} \quad\left(q: \tilde{F}^{\mathbb{C}}(U) \rightarrow \tilde{F}^{\mathbb{C}}(U) / \mathbb{T}_{\mathbb{C}}^{r}\right)
$$


denote the natural projection, where $\tilde{F}(U) / \mathbb{T}_{\mathbb{R}}^{r}\left(\right.$ resp. $\left.\quad \tilde{F}^{\mathbb{C}}(U) / \mathbb{T}_{\mathbb{C}}^{r}\right)$ denotes the orbit space. Note that $\bar{U} \cap \mathbb{R}=I \times\{0\}$, and let

$$
v: \tilde{F}(U) / \mathbb{T}_{\mathbb{R}}^{r} \rightarrow E^{\Sigma}(I, \partial I)
$$

denote the natural map which assigns to an $r$-tuple $\left(f_{1}(z), \cdots, f_{r}(z)\right) \in \tilde{F}(U)$ the $r$-tuple of their configurations represented by their real roots in $I=$ $[-1,1]$. Similarly, note that $\bar{U}=D^{2}$ and let

$$
u: \tilde{F}^{\mathbb{C}}(U) / \mathbb{T}_{\mathbb{C}}^{r} \rightarrow E^{\Sigma}\left(D^{2}, S^{1}\right)
$$

denote the natural map which assigns to an $r$-tuple $\left(f_{1}(z), \cdots, f_{r}(z)\right) \in$ $\tilde{F}^{\mathbb{C}}(U)$ the $r$-tuple of their configurations represented by their roots in $D^{2}$.

(vi) By using this identification $(I, \partial I)=(I \times\{0\}, \partial I \times\{0\}) \subset\left(D^{2}, S^{1}\right)$, we obtain the inclusion map

$$
i_{\Sigma}: E^{\Sigma}(I, \partial I) \stackrel{\subset}{\longrightarrow} E^{\Sigma}\left(D^{2}, S^{1}\right) .
$$

Lemma 8.4. (i) The space $\mathrm{Pol}_{D+\infty}^{\Sigma}$ is simply connected for any $D \in \mathbb{N}^{r}$.

(ii) The map ev $v_{\mathbb{R}}: \tilde{F}(U) \stackrel{\simeq}{\longrightarrow} U\left(\mathcal{K}_{\Sigma}\right)$ is a homotopy equivalence.

(iii) The inclusion map $j_{F}: \tilde{F}^{\mathbb{C}}(U) \stackrel{\simeq}{\longrightarrow} \tilde{F}(U)$ is a homotopy equivalence.

(iv) The map $u: \tilde{F}^{\mathbb{C}}(U) / \mathbb{T}_{\mathbb{C}}^{r} \stackrel{\simeq}{\longrightarrow} E^{\Sigma}\left(D^{2}, S^{1}\right)$ is a homotopy equivalence.

(v) The induced homomorphisms

$$
\left\{\begin{array}{l}
\left(i_{\Sigma}\right)_{*}: \pi_{k}\left(E^{\Sigma}(I, \partial I)\right) \stackrel{\cong}{\longrightarrow} \pi_{k}\left(E^{\Sigma}\left(D^{2}, S^{1}\right)\right) \\
v_{*}: \pi_{k}\left(\tilde{F}(U) / \mathbb{T}_{\mathbb{R}}^{r}\right) \stackrel{\cong}{\longrightarrow} \pi_{k}\left(E^{\Sigma}(I, \partial I)\right)
\end{array}\right.
$$

are isomorphisms for any $k \geq 3$.

Proof. (i) Since $\operatorname{Pol}_{D}^{*}\left(S^{1}, X_{\Sigma}\right)$ is simply connected for any $D \in \mathbb{N}^{r}$ by Proposition 5.9, the space $\mathrm{Pol}_{D+\infty}^{\Sigma}$ is also simply connected.

(ii) Let $i_{0}: U\left(\mathcal{K}_{\Sigma}\right) \rightarrow F(U)$ be the inclusion map given by viewing constants as polynomials. Clearly $e v_{0} \circ i_{0}=$ id. Let $f: F(U) \times[0,1] \rightarrow F(U)$ be the homotopy given by $f\left(\left(f_{1}, \cdots, f_{t}\right), t\right)=\left(f_{1, t}(z), \cdots, f_{r, t}(z)\right)$, where $f_{i, t}(z)=f_{i}(t z)$. This gives a homotopy between $i_{0} \circ e v_{0}$ and the identity map, and this proves that $e v_{0}$ is a deformation retraction. Since $F(U)$ is an infinite dimensional manifold and $\tilde{F}(U)$ is a closed submanifold of $F(U)$ of infinite codimension, it follows from [8, Theorem 2] that the inclusion $\tilde{F}(U) \rightarrow F(U)$ is a homotopy equivalence. Hence the restriction $e v_{\mathbb{R}}$ is also a homotopy equivalence.

(iii) It follows from [20, Lemma 5.4] that the evaluation map $e v=e v_{\mathbb{R}} \circ j_{F}$ : $\tilde{F}^{\mathbb{C}}(U) \stackrel{\simeq}{\longrightarrow} U\left(\mathcal{K}_{\Sigma}\right)$ is a homotopy equivalence. Since $e v_{\mathbb{R}}$ is a homotopy 
equivalence by the assertion (ii), we see that the map $j_{F}: \tilde{F}^{\mathbb{C}}(U) \stackrel{\simeq}{\longrightarrow} \tilde{F}(U)$ is also a homotopy equivalence.

(iv) We know that the map $u$ is a homotopy equivalence by 11, page 133]. However, for the sake of completeness of this paper we will give the another proof. Since all the maps and homotopies which appear in the proof

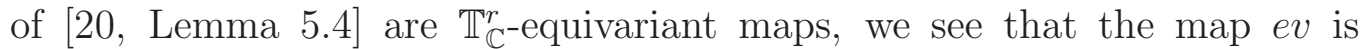

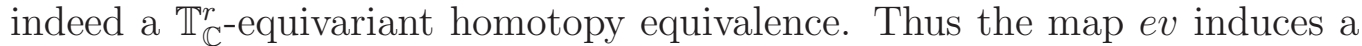
homotopy equivalence $\widetilde{e v}: \tilde{F}^{\mathbb{C}}(U) / \mathbb{T}_{\mathbb{C}}^{r} \stackrel{\simeq}{\longrightarrow} E G \times_{G} U\left(\mathcal{K}_{\Sigma}\right)$, where $G=\mathbb{T}_{\mathbb{C}}^{r}$ and $E G \times{ }_{G} U\left(\mathcal{K}_{\Sigma}\right)$ denotes the Borel construction.

Recall that there is a natural deformation retraction $r t: E G \times{ }_{G} U\left(\mathcal{K}_{\Sigma}\right) \stackrel{\simeq}{\longrightarrow}$ $D J\left(\mathcal{K}_{\Sigma}\right)$ by [3, Theorem 6.29]. If $r_{\Sigma}: E^{\Sigma}\left(D^{2}, S^{1}\right) \stackrel{\simeq}{\longrightarrow} D J\left(\mathcal{K}_{\Sigma}\right)$ denotes the deformation retraction given in [20, Lemma 4.3], then one can check that the following diagram is commutative (up to homotopy equivalence):

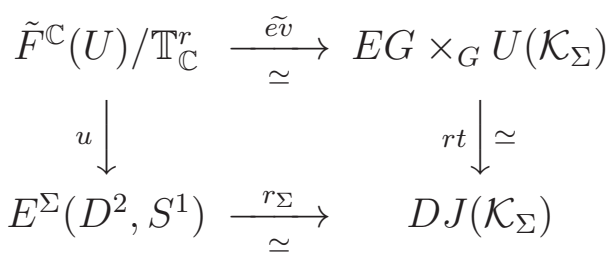

Thus, we see that $u$ is a homotopy equivalence.

(v) Let $p^{\prime}: \tilde{F}(U) / \mathbb{T}_{\mathbb{R}}^{r} \rightarrow \tilde{F}(U) / \mathbb{T}_{\mathbb{C}}^{r}$ be the natural projection. Since $p^{\prime}$ is a bundle projection with fiber $\mathbb{T}_{\mathbb{C}}^{r} / \mathbb{T}_{\mathbb{R}}^{r} \cong\left(S^{1}\right)^{r}$, we see that the map $p^{\prime}$ induces an isomorphism on homotopy groups $\pi_{k}($ ) for any $k \geq 3$. Moreover, since the inclusion $j_{F}$ is a $\mathbb{T}_{\mathbb{C}}^{r}$-equivariant map and the group $\mathbb{T}_{\mathbb{C}}^{r}$ acts freely on both spaces spaces $\tilde{F}^{\mathbb{C}}(U)$ and $\tilde{F}(U)$, there is a map $\tilde{j}_{F}: \tilde{F}^{\mathbb{C}}(U) / \mathbb{T}_{\mathbb{C}}^{r} \rightarrow \tilde{F}(U) / \mathbb{T}_{\mathbb{C}}^{r}$ such that the following diagram is commutative:

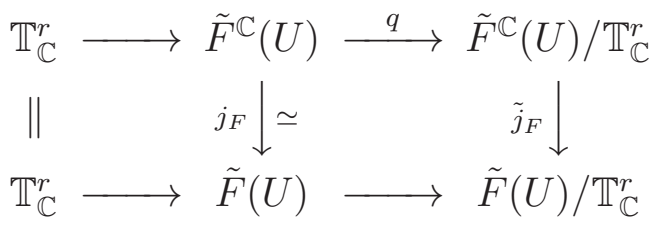

Since two horizontal sequences of (8.7) are fibration sequences and the map $j_{F}$ is a homotopy equivalence, the map $\tilde{j}_{F}$ is a homotopy equivalence. Then if we consider the commutative diagram

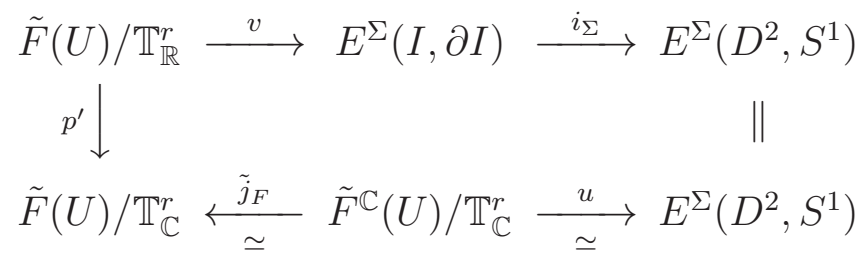


we see that the map $i_{\Sigma}$ induces an epimorphism on homotopy groups $\pi_{k}($ ) for any $k \geq 3$. Let $R: E^{\Sigma}\left(D^{2}, S^{1}\right) \rightarrow E^{\Sigma}(I, \partial I)$ denote the restriction map given by $R\left(\xi_{1}, \cdots, \xi_{r}\right)=\left(\xi_{1} \cap I, \cdots, \xi_{r} \cap I\right)$. Since $R \circ i_{\Sigma}=\mathrm{id}$, the map $i_{\Sigma}$ induces a monomorphism on homotopy groups $\pi_{k}$ ( ) for any $k \geq 1$. Thus the map $i_{\Sigma}$ induces an isomorphism on homotopy groups $\pi_{k}$ ( ) for any $k \geq 3$. By the diagram (8.8), we can easily see that the map $v$ also induces an isomorphism on homotopy groups $\pi_{k}($ ) for any $k \geq 3$.

Proof of Theorem 8.2. Since $\operatorname{Pol}_{D+\infty}^{\Sigma}$ and $\Omega U\left(\mathcal{K}_{\Sigma}\right)$ are simply connected, it suffices to show that

$$
\left(j_{D+\infty}\right)_{*}: \pi_{k}\left(\operatorname{Pol}_{D+\infty}^{\Sigma}\right) \stackrel{\cong}{\longrightarrow} \pi_{k}\left(\Omega U\left(\mathcal{K}_{\Sigma}\right)\right)
$$

is an isomorphism for any $k \geq 2$. Let us identify $\mathbb{C}=\mathbb{R}^{2}$ and let $U=$ $(-1,1) \times(-1,1)$ as before. Let scan $: \tilde{F}(\mathbb{C}) \rightarrow \operatorname{Map}(\mathbb{R}, \tilde{F}(U))$ denote the map given by $\operatorname{scan}\left(f_{1}(z), \cdots, f_{r}(z)\right)(w)=\left(f_{1}(z+w), \cdots, f_{r}(z+w)\right)$ for $w \in \mathbb{R}$, and consider the diagram

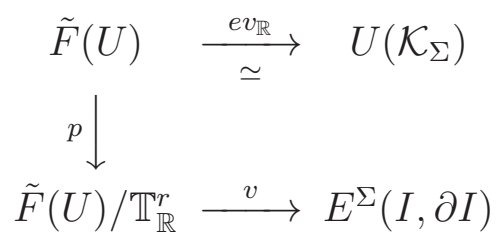

This induces the commutative diagram below

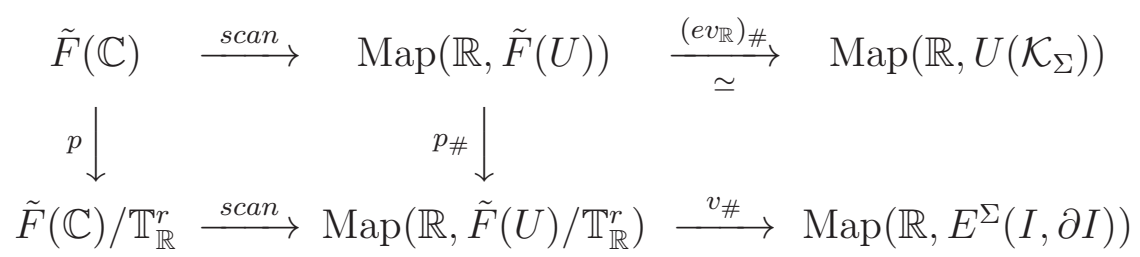

Observe that $\operatorname{Map}(\mathbb{R}, \cdot)$ can be replaced by $\operatorname{Map}^{*}\left(S^{1}, \cdot\right)$ by extending from $\mathbb{R}$ to $S^{1}=\mathbb{R} \cup \infty$ (as base-point preserving maps). Thus by setting

$$
\left\{\begin{array}{l}
\widehat{j_{D}}: \operatorname{Pol}_{D}^{*}\left(S^{1}, X_{\Sigma}\right) \stackrel{\subset}{\longrightarrow} \tilde{F}(\mathbb{C}) \stackrel{\text { scan }}{\longrightarrow} \operatorname{Map}^{*}\left(S^{1}, \tilde{F}(U)\right)=\Omega \tilde{F}(U) \\
\widehat{j_{D}^{\prime}}: E_{D}^{\Sigma, \mathbb{R}}(\mathbb{C}) \stackrel{\subset}{\longrightarrow} \tilde{F}(\mathbb{C}) \stackrel{\text { scan }}{\longrightarrow} \operatorname{Map}^{*}\left(S^{1}, \tilde{F}(U) / \mathbb{T}_{\mathbb{R}}^{r}\right)=\Omega \tilde{F}(U) / \mathbb{T}_{\mathbb{R}}^{r}
\end{array}\right.
$$

we obtain the following commutative diagram

$$
\begin{array}{ccc}
\operatorname{Pol}_{D}^{*}\left(S^{1}, X_{\Sigma}\right) \stackrel{\widehat{j_{D}}}{\longrightarrow} \quad \Omega \tilde{F}(U) \stackrel{\Omega e v_{\mathbb{R}}}{\simeq} \Omega U\left(\mathcal{K}_{\Sigma}\right) \\
\cong \downarrow \\
E_{D}^{\Sigma, \mathbb{R}}(\mathbb{C}) \stackrel{\widehat{j_{D}^{\prime}}}{\longrightarrow} \Omega \tilde{F}(U) / \mathbb{T}_{\mathbb{R}}^{r} \stackrel{\Omega v}{\longrightarrow} \Omega E^{\Sigma}(I, \partial I)
\end{array}
$$


Since $p$ is a covering projection, $\Omega p$ is a homotopy equivalence. If we identify $\mathrm{Pol}_{D+\infty}^{\Sigma}$ with the colimit $\lim _{t \rightarrow \infty} E_{D+t \boldsymbol{e}}^{\Sigma, \mathbb{R}}(\mathbb{C})$, by replacing $D$ by $D+t \boldsymbol{e}(t \in \mathbb{N})$ and letting $t \rightarrow \infty$, we obtain the following homotopy commutative diagram:

$$
\begin{array}{cc}
\operatorname{Pol}_{D+\infty}^{\Sigma} \stackrel{\widehat{j_{D+\infty}}}{\longrightarrow} \quad \Omega \tilde{F}(U) \stackrel{\operatorname{ev}_{\mathbb{R}}}{\longrightarrow} \Omega U\left(\mathcal{K}_{\Sigma}\right) \\
\| \quad \Omega p \downarrow \simeq \\
\operatorname{Pol}_{D+\infty}^{\Sigma} \stackrel{\widehat{j_{D+\infty}^{\prime}}}{\longrightarrow} \Omega \tilde{F}(U) / \mathbb{T}_{\mathbb{R}}^{r} \stackrel{\Omega v}{\longrightarrow} \Omega E^{\Sigma}(I, \partial I)
\end{array}
$$

where we set $\widehat{j_{D+\infty}}=\lim _{t \rightarrow \infty} \widehat{j_{D+t e}}$ and $\widehat{j_{D+\infty}^{\prime}}=\lim _{t \rightarrow \infty} \widehat{j_{D+t e}^{\prime}}$.

Since $\left(\Omega e v_{\mathbb{R}}\right) \circ \widehat{j_{D+t e}}=j_{D+t e}$ and $(\Omega v) \circ \widehat{j_{D+t e}^{\prime}}=s c_{D+t e}$ (up to homotopy equivalence), we also obtain the following two equalities:

$$
j_{D+\infty}=\left(\Omega e v_{\mathbb{R}}\right) \circ \widehat{j_{D+\infty}}, \quad S^{H}=(\Omega v) \circ \widehat{j_{D+\infty}^{\prime}} .
$$

Since the map $e v_{\mathbb{R}}$ is a homotopy equivalence, it suffices to prove that

$$
\left(\widehat{j_{D+\infty}}\right)_{*}: \pi_{k}\left(\mathrm{Pol}_{D+\infty}^{\Sigma}\right) \stackrel{\cong}{\longrightarrow} \pi_{k}(\Omega \tilde{F}(U))
$$

is an isomorphism for any $k \geq 2$. Since $S^{H}=(\Omega v) \circ \widehat{j_{D+\infty}^{\prime}}$ is a homotopy equivalence and the map $\Omega v$ induces an isomorphism on homotopy groups $\pi_{k}$ ( ) for any $k \geq 2$ (by (v) of Lemma 8.4), by the diagram (8.10) we see that the map $\widehat{j_{D+\infty}}$ induces an isomorphism on homotopy groups $\pi_{k}(\mathrm{)}$ for any $k \geq 2$. This completes the proof of Theorem 8.2 .

Corollary 8.5. Let $X_{\Sigma}$ be a simply connected non-singular toric variety such that the condition 2.14.1) is satisfied.

(i) The two-fold loop map $\Omega^{2} v: \Omega^{2} \tilde{F}(U) / \mathbb{T}_{\mathbb{R}}^{r} \stackrel{\simeq}{\longrightarrow} \Omega^{2} E^{\Sigma}(I, \partial I)$ is a homotopy equivalence.

(ii) The loop map $\Omega i_{\Sigma}: \Omega E^{\Sigma}(I, \partial I) \rightarrow \Omega E^{\Sigma}\left(D^{2}, S^{1}\right)$ is a universal covering projection with fiber $\mathbb{Z}^{r}$ (up to homotopy equivalence).

Proof. (i) The assertion (i) follows from (v) of Lemma 8.4 .

(ii) Since $\Omega^{2} i_{\Sigma}$ is a homotopy equivalence (by (v) of Lemma 8.4), the assertion (ii) easily follows from the following two equalities:

$$
\pi_{1}\left(\Omega E^{\Sigma}(I, \partial I)\right)=0, \quad \pi_{1}\left(\Omega E^{\Sigma}\left(D^{2}, S^{1}\right)\right)=\mathbb{Z}^{r} .
$$

Since $\operatorname{Pol}_{D+\infty}^{\Sigma}$ is simply connected (by (i) of Lemma 8.4), by Theorem 8.2 we have an isomorphism $0=\pi_{1}\left(\operatorname{Pol}_{D+\infty}^{\Sigma}\right) \cong \pi_{1}\left(\Omega E^{\Sigma}(I, \partial I)\right)$ and the first 
equality of (8.12) holds. It remains the second isomorphism in (8.12). Since there is a homotopy equivalence $E^{\Sigma}\left(D^{2}, S^{1}\right) \simeq D J\left(\mathcal{K}_{\Sigma}\right)$ ([20, Lemma 4.3]), it suffices to show that there is an isomorphism $\pi_{2}\left(D J\left(\mathcal{K}_{\Sigma}\right)\right) \cong \mathbb{Z}^{r}$. Since $X_{\Sigma}$ is simply connected and $\pi_{2}\left(X_{\Sigma}\right)=\mathbb{Z}^{r-n}$ (by $(\underline{2.26})$ ), it follows from the homotopy exact sequence induced from (3.5) that there is a short exact sequence $0 \rightarrow \pi_{2}\left(X_{\Sigma}\right)=\mathbb{Z}^{r-n} \rightarrow \pi_{2}\left(D J\left(\mathcal{K}_{\Sigma}\right)\right) \stackrel{\partial}{\longrightarrow} \pi_{1}\left(\mathbb{T}_{\mathbb{C}}^{n}\right)=\mathbb{Z}^{n} \rightarrow 0$. Thus, we have an isomorphism $\pi_{2}\left(D J\left(\mathcal{K}_{\Sigma}\right)\right) \cong \mathbb{Z}^{r}$.

\section{Proofs of the main results}

In this section we give the proofs of the main results (Theorem 2.16, Corollary 2.17, Theorem 2.20, Corollary 2.21, Corollary 2.18, Corollary 2.22).

Proofs of Theorem 2.16 and Theorem 2.20. If $\sum_{k=1}^{r} d_{k} \boldsymbol{n}_{k}=\mathbf{0}_{n}$, Theorem 2.16 easily follows from Theorem 6.5 and Theorem 8.2. Next assume that $\sum_{k=1}^{r} d_{k} \boldsymbol{n}_{k} \neq \mathbf{0}_{n}$. It follows from the assumption (2.14.1) that there is an $r$-tuple $D_{*}=\left(d_{1}^{*}, \cdots, d_{r}^{*}\right) \in \mathbb{N}^{r}$ such that $\sum_{k=1}^{r} d_{k}^{*} \boldsymbol{n}_{k}=\mathbf{0}_{n}$. If we choose a sufficiently large integer $m_{0} \in \mathbb{N}$, then the condition $d_{k}<m_{0} d_{k}^{*}$ holds for each $1 \leq k \leq r$. Then note that $j_{D}: \operatorname{Pol}_{D}^{*}\left(S^{1}, X_{\Sigma}\right) \rightarrow \Omega \mathcal{Z}_{\mathcal{K}_{\Sigma}}$ is given by the composite of maps

$$
j_{D}=j_{D_{0}} \circ s_{D, D_{0}}: \operatorname{Pol}_{D}^{*}\left(S^{1}, X_{\Sigma}\right) \stackrel{s_{D, D_{0}}}{\longrightarrow} \mathrm{Pol}_{D_{0}}^{*}\left(S^{1}, X_{\Sigma}\right) \stackrel{j_{D_{0}}}{\longrightarrow} \Omega \mathcal{Z}_{\mathcal{K}_{\Sigma}}
$$

as in (6.17), where $D_{0}=m_{0} D_{*}=\left(m_{0} d_{1}^{*}, m_{0} d_{2}^{*}, \cdots, m_{0} d_{r}^{*}\right)$. Since the maps $s_{D, D_{0}}$ and $j_{D_{0}}$ are homotopy equivalences through dimension $d(D, \Sigma)$ and $d\left(D_{0}, \Sigma\right)$ (by Theorem 6.6 and Theorem 2.16), by using $d(D, \Sigma) \leq d\left(D_{0}, \Sigma\right)$ the map $j_{D}$ is a homotopy equivalence through dimension $d(D, \Sigma)$ and Theorem 2.20 follows.

Proof of Corollary 2.17. Since $\Omega q_{\Sigma}: \Omega \mathcal{Z}_{\mathcal{K}_{\Sigma}} \rightarrow \Omega X_{\Sigma}$ is a universal covering, the assertion follows from (2.23) and Theorem 2.16 .

Proofs of Corollary [2.21. Consider the map $i_{D}: \operatorname{Pol}_{D}^{*}\left(S^{1}, X_{\Sigma}\right) \rightarrow \Omega X_{\Sigma}$ defined by the composite of maps

$$
i_{D}=\Omega q_{\Sigma} \circ j_{D}
$$

where $j_{D}: \operatorname{Pol}_{D}^{*}\left(S^{1}, X_{\Sigma}\right) \rightarrow \Omega \mathcal{Z}_{\mathcal{K}_{\Sigma}}$ denotes the map given by (6.17). Since $\Omega q_{\Sigma}: \Omega \mathcal{Z}_{\mathcal{K}_{\Sigma}} \rightarrow \Omega X_{\Sigma}$ is a universal covering, the assertion follows from Theorem 2.20 . 
Proofs of Corollary 2.18 and Corollary 2.22. If $X_{\Sigma}$ is compact and $\Sigma(1) \subset$ $\Sigma_{1} \varsubsetneqq \Sigma$, the condition (2.14.1) holds for the fan $\Sigma_{1}$. Thus, Corollary 2.18 follows from Theorem 2.16 and Corollary 2.17. Similarly, Corollary 2.22 also follows from Theorem 2.20 and Corollary 2.21.

Acknowledgements. The second author was supported by JSPS KAKENHI Grant Number 18K03295. This work was also supported by the Research Institute for Mathematical Sciences, a Joint Usage/Research Center located in Kyoto University.

\section{References}

[1] M. Adamaszek, A. Kozlowski and K. Yamaguchi, Spaces of algebraic and continuous maps between real algebraic varieties, Quart. J. Math. 62 (2011), 771-790.

[2] M. F. Atiyah and J. D. S. Jones, Topological aspects of Yang-Mills theory, Commun. Math. Phys. 59 (1978), 97-118.

[3] V. M. Buchstaber and T. E. Panov, Torus actions and their applications in topology and combinatorics, Univ. Lecture Note Series 24, Amer. Math. Soc. Providence, 2002.

[4] R. L. Cohen, J. D. S. Jones and G. Segal, Stability for holomorphic spheres and Morse theory, Contemporary Math., 258 (2000), 87-106.

[5] D. A. Cox, The homogenous coordinate ring of a toric variety, J. Algebraic Geometry 4 (1995), 17-50.

[6] D. A. Cox, The functor of a smooth toric variety, Tohoku Math. J. 47 (1995), 251-262.

[7] D. A. Cox, J. B. Little and H. K. Schenck, Toric varieties, Graduate Studies in Math. 124, Amer. Math. Soc., 2011.

[8] J. Eells, Jr. and N. H. Kuiper, Homotopy negligible subsets, Compositio Math., 21, (1969), 155-161.

[9] M. Gromov, Oka's principle for holomorphic sections of elliptic bundles, J. Amer. Math. Soc. 2 (1989) 851-897.

[10] M. A. Guest, Instantons, rational maps and harmonic maps, Methamatica Contemporanea 2 (1992), 113-155. 
[11] M. A. Guest, The topology of the space of rational curves on a toric variety, Acta Math. 174 (1995), 119-145.

[12] M.A. Guest, A. Kozlowski and K. Yamaguchi, The topology of spaces of coprime polynomials, Math. Z. 217 (1994), 435-446.

[13] M. A. Guest, A. Kozlowski and K. Yamaguchi, Spaces of polynomials with roots of bounded multiplicity, Fund. Math. 116 (1999), 93-117.

[14] A. Kozlowski, M. Ohno and K. Yamaguchi, Spaces of algebraic maps from real projective spaces to toric varieties, J. Math. Soc. Japan 68 (2016), 745-771

[15] A. Kozlowski and K. Yamaguchi, Topology of complements of discriminants and resultants, J. Math. Soc. Japan 52 (2000), 949-959.

[16] A. Kozlowski and K. Yamaguchi, Simplicial resolutions and spaces of algebraic maps between real projective spaces, Topology Appl. 160 (2013), $87-98$.

[17] A. Kozlowski and K. Yamaguchi, The homotopy type of spaces of coprime polynomials revisited, Topology Appl. 206 (2016), 284-304.

[18] A. Kozlowski and K. Yamaguchi, The homotopy type of spaces of polynomials with bounded multiplicity, Publ. RIMS. Kyoto Univ., 52 (2016), 297-308.

[19] A. Kozlowski and K. Yamaguchi, The homotopy type of spaces of resultants of bounded multiplicity, Topology Appl. 232 (2017), 112-139.

[20] A. Kozlowski and K. Yamaguchi, The homotopy type of spaces of rational curves on a toric variety, Topology Appl. 249 (2018), 19-42.

[21] J. Mostovoy, Spaces of rational loops on real projective space, Trans. Amer. Math. Soc. 35 (2001), 1951-1970-293.

[22] J. Mostovoy, Spaces of rational maps and the Stone-Weierstrass Theorem, Topology 45 (2006), 281-293.

[23] J. Mostovoy, Truncated simplicial resolutions and spaces of rational maps, Quart. J. Math. 63 (2012), 181-187.

[24] J. Mostovoy and E. Munguia-Villanueva, Spaces of morphisms from a projective space to a toric variety, Rev. Colombiana Mat. 48 (2014), 41-53. 
[25] T. E. Panov, Geometric structures on moment-angle manifolds, Russian Math. Surveys 68 (2013), 503-568.

[26] G. B. Segal, The topology of spaces of rational functions, Acta Math. 143 (1979), 39-72.

[27] V. A. Vassiliev, Complements of discriminants of smooth maps, Topology and Applications, Amer. Math. Soc., Translations of Math. Monographs 98, 1992 (revised edition 1994).

[28] V. A. Vassiliev, Topologia dopolneniy k diskriminantam, Fazis, Moskva 1997. 